\title{
EQUIVARIANT DE RHAM THEORY AND GRAPHS*
}

\author{
V. GUILLEMIN ${ }^{\dagger}$ AND C. ZARA Z $^{\ddagger}$
}

\begin{abstract}
Goresky, Kottwitz and MacPherson have recently shown that the computation of the equivariant cohomology ring of a $G$-manifold can be reduced to a computation in graph theory. This opens up the possibility that many of the fundamental theorems in equivariant de Rham theory may, on closer inspection, turn out simply to be theorems about graphs. In this paper we show that for some familiar theorems, this is indeed the case.
\end{abstract}

Introduction. This article will consist of two essentially disjoint parts. Part 1 is an exposition of (mostly) well-known results about $G$-manifolds. In section 1.1-1.3 we review the definition of the equivariant de Rham cohomology ring of a $G$-manifold and recall the statements of the two fundamental "localization theorems" in equivariant de Rham theory: the Atiyah-Bott-Berline-Vergne theorem and the Jeffrey-Kirwan theorem. In section 1.4 we discuss the "Smith" problem for $G$-manifolds (which is concerned with the question: Given a $G$-manifold with isolated fixed points, what kinds of representations can occur as isotropy representations at the fixed points?) Then in sections 1.5-1.7 we report on some very exciting recent results of GoreskyKottwitz-MacPherson which have to do with the tie-in between "equivariant de Rham theory" and "graphs" alluded to in our title. These results show that for a large class of $G$-manifolds, $M$, with $M^{G}$ finite, the equivariant cohomology ring of $M$ is isomorphic to the equivariant cohomology ring of a pair $(\Gamma, \alpha)$, where $\Gamma$ is the intersection graph of a necklace of embedded $S^{2}$ 's, each of which is equipped with a circle action (i.e., an axis of symmetry), and $\alpha$ is an "axial" function which describes the directions in which the axes of these $S^{2}$ 's are tilted. Finally, in section 1.8 we discuss a Morse theoretic recipe for computing the Betti numbers of $M$ in terms of the pair $(\Gamma, \alpha)$.

The second part of this article is concerned with the combinatorial invariants of a pair $(\Gamma, \alpha), \Gamma$ being any finite simple $d$-valent graph and $\alpha$ an abstract analogue of the axial function alluded to above. In particular, for such a pair we will prove combinatorial versions of the theorems described in sections 1.2-1.3 and 1.8. These combinatorial "localization" theorems help to shed some light on the role of the localization theorems in Smith theory : From the localization theorems one can generate a lot of complicated identities among the weights of the isotropy representations. However, the question of whether one can extract from these identities any new information about the isotropy representations themselves has been an open question for a long time. Our graph theoretical results seem to indicate that one can't.

This article is the first of a series of two articles on graphs and equivariant cohomology. In the second article in this series we will discuss K-theoretical analogues of the results above and give a purely combinatorial proof of the so-called "quantization commutes with reduction" conjecture.

1.1. Equivariant de Rham theory. Let $G$ be an $n$-dimensional Lie group which is compact, connected and abelian, i.e., an $n$-dimensional torus. Let $\mathfrak{g}$ be its

*Received November 11, 1998; accepted for publication November 30, 1998.

† Department of Mathematics, MIT, Cambridge, MA 02139 (vwg@math.mit.edu). Research Supported in part by NSF grant DMS 890771.

${ }^{\ddagger}$ Department of Mathematics, MIT, Cambridge, MA 02139 (czara@math.mit.edu). 
Lie algebra and $\mathfrak{g}^{*}$ the vector space dual of $\mathfrak{g}$. We will fix a basis $\xi^{1}, \ldots, \xi^{n}$ of $\mathfrak{g}$ and let $x_{1}, \ldots, x_{n}$ be the dual basis. Using this basis, the symmetric algebra $S\left(\mathfrak{g}^{*}\right)$ can be identified with the polynomial ring $\mathbb{C}\left[x_{1}, \ldots, x_{n}\right]$.

Let $M$ be a $2 d$-dimensional manifold and $\tau$ an action of $G$ on $M$. From $\tau$ one gets an infinitesimal action, $\partial \tau$, of $\mathfrak{g}$ on $M$ which associates to every element $\xi$ of $\mathfrak{g}$ a vector field $\xi_{M}$. Let $\Omega(M)$ be the usual complex of de Rham forms on $M$ and $\Omega(M)^{G}$ the subcomplex of $G$-invariant de Rham forms. One defines the equivariant de Rham complex of $M$ to be the tensor product

$$
\Omega_{G}(M)=\Omega(M)^{G} \otimes S\left(\mathfrak{g}^{*}\right)
$$

with the coboundary operator

$$
d_{G}(\omega \otimes f)=d \omega \otimes f+\sum \iota\left(\xi_{M}^{i}\right) \omega \otimes x_{i} f .
$$

The equivariant cohomology ring of $M, H_{G}(M)$, is the cohomology ring of this complex. A few properties of this ring which we will need below are:

1. $H_{G}(M)$ is an $S\left(\mathfrak{g}^{*}\right)$-module. (This follows from the fact that $\Omega_{G}(M)$ is an $S\left(\mathfrak{g}^{*}\right)$ module by (1.1) and $d_{G}$ is an $S\left(\mathfrak{g}^{*}\right)$ module morphism by (1.2).)

2. $H_{G}(p t)=S\left(\mathfrak{g}^{*}\right)$.

3. Suppose $M$ is compact and oriented. Then there is an integration operation

$$
\int: \Omega_{G}(M) \longrightarrow S\left(\mathfrak{g}^{*}\right)
$$

defined by

$$
\int(\omega \otimes f)=f \int \omega
$$

It is easily checked that $\int d_{G}=0$ and hence that this integration operation induces an integration operation on cohomology

$$
\int: H_{G}(M) \longrightarrow S\left(\mathfrak{g}^{*}\right)
$$

4. One can write $d_{G}$ as a sum, $d_{1}+d_{2}, d_{1}$ and $d_{2}$ being the first and second terms on the right hand side of (1.2). Thus $\Omega_{G}(M)$ is a bi-complex, and the additive structure of $H_{G}(M)$ can be computed by the spectral sequence of this bi-complex. The $E_{1}$ term in this spectral sequence is the $d_{1}$-cohomology of $\Omega_{G}(M)$, namely

$$
H(M) \otimes S\left(\mathfrak{g}^{*}\right)
$$

One says that $M$ is equivariantly formal if the spectral sequence is trivial, i.e. if, as vector spaces,

$$
H_{G}(M)=H(M) \otimes S\left(\mathfrak{g}^{*}\right) .
$$

One can show, by the way, that if (1.6) holds as an identity of vector spaces, it also holds as an identity of $S\left(\mathfrak{g}^{*}\right)$-modules. However, (1.6) doesn't, in general, tell one very much about the ring structure of $H_{G}(M)$ (about which we will have more to say in $§ 1.6)$. 
The property of being equivariantly formal is a bit technical; however there are a number of interesting assumptions on $M$ which will imply this property. (See [GKM].) Of these assumptions the one that will be of most interest to us is the following:

THEOREM(Kirwan). If $M$ is a symplectic manifold and the action $\tau$ is Hamiltonian, $M$ is equivariantly formal.

1.2. The Atiyah-Bott-Berline-Vergne localization theorem. Let $M$ be compact and oriented and, also, to simplify the statement of the localization theorem, let $M^{G}$ be finite. For $p \in M^{G}$ one has an isotropy representation $\tau_{p}$ of $G$ on $T_{p}$ and we will denote the weights of this representation by $\alpha_{i, p}, i=1, \ldots, d$. Since $\tau_{p}$ is a real representation, these weights are, strictly speaking, only defined up to sign; however, since $M$ is oriented, the product

$$
\alpha_{1, p} \ldots \alpha_{d, p}
$$

is well-defined as an element of $S^{d}\left(\mathfrak{g}^{*}\right)$. Let

$$
j_{p}: p t \longrightarrow M
$$

be the mapping " $p t$ " onto $p$ and note that if $c$ is in $H_{G}(M), j_{p}^{*} c$ is in $H_{G}(p t)$ and thus in $S\left(\mathfrak{g}^{*}\right)$. The localization theorem asserts that, for every equivariant cohomology class $c \in H_{G}(M)$,

$$
\int c=\sum_{p \in M^{G}} \frac{j_{p}^{*} c}{\prod \alpha_{i, p}}
$$

There are many deep and beautiful applications of (1.7) but the focus of our interest in this article is that (1.7) implies a lot of complicated identities among the weights $\alpha_{i, p}$. For instance, for $c=1$, it implies

$$
\sum\left(\prod \alpha_{i, p}\right)^{-1}=0 \text {. }
$$

What are these identities? In particular, are there simpler identities of which they are formal consequences? We will show in part 2 of this article that one can shed some light on these questions by looking at a graph-theoretical analogue of (1.7).

1.3. The Jeffrey-Kirwan theorem. Another interesting source for identities of type (1.7) is the Jeffrey-Kirwan theorem ([JK]): Let $K$ be a one-dimensional connected closed subgroup of $G$ with Lie algebra $\mathfrak{k}$ and let $\xi \in \mathfrak{k}$ be a basis vector of the group lattice of $K$. Suppose $M$ possesses a $G$-invariant symplectic form $\omega$ and that the action of $K$ on $M$ is Hamiltonian, i.e.

$$
\iota\left(\xi_{M}\right) \omega=-d f
$$

$f$ being a $G$-invariant function. In addition suppose that

$$
M^{K}=M^{G}
$$

and hence that the critical points of $f$ coincide with the fixed points of $G$. Let $a$ be a regular value of $f$ and let $M_{a}=f^{-1}(a)$. By the remark above, $M_{a}$ contains no $K$-fixed points, so the action of $K$ on $M_{a}$ is locally free and the quotient space

$$
M_{a} / K=: M_{\text {red }}
$$


is an orbifold. Moreover, from the action of $G$ on $M_{a}$ one gets an inherited action of the quotient group

$$
G / K=: G_{1}
$$

on $M_{\text {red }}$. Let $j$ be the inclusion of $M_{a}$ into $M$ and $\pi$ the projection of $M_{a}$ onto $M_{\text {red }}$. By the Marsden-Weinstein theorem there exists a symplectic form $\omega_{\text {red }}$ on $M_{r e d}$ satisfying $\pi^{*} \omega_{r e d}=j^{*} \omega$. In particular, $M_{r e d}$ is oriented. If $\mathfrak{g}_{1}$ is the Lie algebra of $G_{1}$, its vector space dual, $\mathfrak{g}_{1}^{*}$, can be identified with the annihilator $\mathfrak{g}_{\xi}^{*}$ of $\xi$ in $\mathfrak{g}$; so there is an integration operator

$$
\int: H_{G_{1}}\left(M_{r e d}\right) \longrightarrow S\left(\mathfrak{g}_{\xi}^{*}\right)
$$

Also, since the action of $K$ on $M_{a}$ is locally free, the map $\pi$ induces an isomorphism

$$
\pi^{*}: H_{G_{1}}\left(M_{r e d}\right) \longrightarrow H_{G}\left(M_{a}\right)
$$

so one gets a map

$$
\left(\pi^{*}\right)^{-1} j^{*}=: \mathcal{K}
$$

of $H_{G}(M)$ into $H_{G_{1}}\left(M_{r e d}\right)$. The Jeffrey-Kirwan theorem asserts that for every equivariant cohomology class $c \in H_{G}(M)$,

$$
\int \mathcal{K}(c)=\operatorname{Res}_{\xi}\left(\sum_{f(p)>a} \frac{j_{p}^{*} c}{\prod \alpha_{j, p}}\right), \quad p \in M^{G},
$$

$\operatorname{Res}_{\xi}$ being the residue of the rational function in brackets with respect to the " $\xi$ coordinate" on $\mathfrak{g}^{*}$, the other coordinates being held fixed. (This residue can be defined intrinsically to be an element of $S\left(\mathfrak{g}_{\xi}^{*}\right)$. See $\left.\S 2.6\right)$

1.4. The Smith problem. The Smith conjecture asserts that if $M^{G}$ consists of two points, $p$ and $q$, the isotropy representation of $G$ at $p$ is isomorphic (as a representation over $\mathbb{R}$ ) to the isotropy representation of $G$ at $q$. The first complete proof of this theorem (for $G$ an arbitrary compact Lie group) is due to Atiyah, Bott and Milnor (See [AB2], Theorem 3.83). If the cardinality of $M^{G}$ is greater than two, the question of how the isotropy representations of $G$ at distinct fixed points are related to each other is still open and is known as the "Smith problem". In this section we will describe a few of the more obvious relations:

1. Relations of type $J$.

Suppose that $M$ admits a $G$-invariant almost-complex structure. Then for every $p \in M^{G}$, the isotropy representations of $G$ on $T_{p}$ is a complex representation, so the weights of this representation,

$$
\alpha_{i, p}, \quad i=1, \ldots, d
$$

are unambiguously defined (not just defined up to sign).

For every closed subgroup $H$ of $G$ let $\mathfrak{h}$ be the Lie algebra of $H$ and

$$
\rho_{H}: \mathfrak{g}^{*} \longrightarrow \mathfrak{h}^{*}
$$


the transpose of the inclusion map $\mathfrak{h} \longrightarrow \mathfrak{g}$. Let $E$ be a connected component of $M^{H}$ and $p$ and $q$ elements of $E^{G}$. We claim that the weights (1.12) can be ordered so that

$$
\rho_{H} \alpha_{i, p}=\rho_{H} \alpha_{i, q} .
$$

Proof. Let $x$ be an arbitrary point of $E$ and consider the isotropy representation of $H$ on its normal space to $E$ at $x$. This representation is a complex representation, so the weights of this representation are unambiguously defined and can't vary as $x$ varies in $E$. Thus, in particular, they have to be the same at $p$ and at $q$,implying (1.13).

2. Relations of type $\omega$.

Assuming that $M$ admits a $G$-invariant almost-complex structure, $J$, is equivalent to assuming that $M$ admits a $G$-invariant "almost-symplectic" structure, i.e., a two-form, $\omega$, which is everywhere of maximal rank. Suppose that $\omega$ is actually a symplectic form and the action $\tau$ is Hamiltonian, or, in other words, that there exists a moment map

$$
\Phi: M \longrightarrow \mathfrak{g}^{*} .
$$

From the convexity theorem ( $[\mathrm{A}],[\mathrm{GS}])$ one gets

THEOREM. Let $\Delta$ be the set of regular values of $\Phi$ in $\Phi(M)$. Then $\Delta$ is a disjoint union of open convex polytopes. Moreover, the vertices of these polytopes are the images of the fixed points $p \in M^{G}$ and the edges going out of these vertices are pointing in the directions of the vectors $\alpha_{i, p}$.

The one-skeleton, $\Gamma$, of this configuration is called the moment graph of $M$. (See [Gu].) It exhibits a lot of relations among the $\alpha_{i, p}$ 's which are probably not much simpler than the relations (1.7) but have the virtue of being of a more geometric character.

1.5. The Goresky-Kottwitz-MacPherson graph. We will assume from now on that $M$ admits a $G$-invariant almost-complex structure. Thus, for very $p \in M^{G}$, the weights, $\alpha_{i, p} \in \mathfrak{g}^{*}$, are unambiguously defined. In addition we will assume: if $i \neq j$, $\alpha_{i, p}$ and $\alpha_{j, p}$ are linearly independent. This "GKM hypothesis" has the following consequence: Let $\mathfrak{h}=\mathfrak{h}_{i}$ be the annihilator of $\alpha_{i, p}$ in $\mathfrak{g}$ and let $H$ be the $(n-1)$ dimensional subtorus of $G$ with Lie algebra, $\mathfrak{h}$. Clearly, $p \in M^{G} \subseteq M^{H}$.

Proposition. Let $E$ be the connected component of $M^{H}$ containing $p$. Then

$$
E \cong \mathbb{C} P^{1} \cong S^{2}
$$

and the action of $G$ on $E$ is the standard action of the circle, $S^{1}$, on $S^{2}$ by "rotation about the z-axis". In particular $E$ contains just two $G$-fixed points (one of which is p).

Proof. The tangent space to $E$ at $p$ is the 2-dimensional subspace of $T_{p}$ on which $G$ acts with weight $\alpha_{i, p}$ so $E$ itself is 2-dimensional. Since $E$ is compact and the action of $G / H$ is non-trivial, $E$ is diffeomorphic to $S^{2}$, and this action is the standard action of $S^{1}$ on $S^{2}$ by the Korn-Lichtenstein theorem. 
Let $q$ be the other fixed point of $G$ in $E$. Let $\alpha_{p, e}=\alpha_{i, p}$ be the weight of the isotropy representation of $G$ on $T_{p} E$ and let $\alpha_{q, e}=\alpha_{j, q}$ be the weight of the isotropy representation of $G$ on $T_{q} E$. From the fact that the action of $G$ on $E$ is diffeomorphic to the standard action of $S^{1}$ on $S^{2}$ it follows that

$$
\alpha_{p, e}=-\alpha_{q, e}
$$

For each of the weights $\alpha_{i, p}$ one gets an embedded $\mathbb{C} P^{1}$ of the type above, and we can represent these $\mathbb{C} P^{1}$ 's graphically by $d$ lines issuing from $p$. Each of these lines joins $p$ to another fixed point, $q$, and the $\mathbb{C} P^{1}$ 's associated with the weights, $\alpha_{j, q}$, can also be represented graphically by $d$ lines issuing from $q$. One of these will be the line from $p$ to $q$, but the remaining $d-1$ lines will join $q$ to other fixed points. By repeating this construction over and over until one runs out of fixed points, one obtains a finite $d$-valent graph, $\Gamma$, the vertices of which are the fixed points of $G$ and the edges of which correspond to embedded $\mathbb{C} P^{1}$ 's, each of these $\mathbb{C} P^{1}$ 's being a connected component of the fixed point set of an $(n-1)$-dimensional subtorus of $G$. We will call $\Gamma$ the Goresky-Kottwitz-MacPherson (GKM) graph of $M$.

Example. Suppose $M$ is a Hamiltonian $G$-manifold whose moment map, $\Phi$, maps $M^{G}$ injectively into $\mathfrak{g}^{*}$. Then $\Phi$ embeds the GKM graph into $\mathfrak{g}^{*}$, and its image is the moment graph.

1.6. The Goresky-Kottwitz-MacPherson theorem. The graph $\Gamma$ is equipped with an additional piece of structure. Namely let $I_{\Gamma}$ be the incidence relation of this graph: the set of all pairs $(p, e), p$ being a vertex and $e$ an edge containing p. Then one has a map

$$
\alpha: I_{\Gamma} \longrightarrow \mathfrak{g}^{*}-\{0\}
$$

mapping $(p, e)$ to the weight $\alpha_{p, e}$. We will call this the axial function of $\Gamma$. It has the following properties (the first two of which we have already commented on):

1. If $e$ is an edge and $p$ and $q$ are the vertices joined by $e$

$$
\alpha_{p, e}=-\alpha_{q, e} .
$$

2. If $p$ is a vertex and $e_{1}, \ldots, e_{d}$ are the edges containing $p$, the vectors

$$
\alpha_{p, e_{i}}, \quad i=1, \ldots, d
$$

are pair-wise linearly independent.

3. Let $e$ be an edge and $p$ and $q$ the vertices joined by $e$. Let

$$
\mathfrak{g}_{e}=\left\{\xi \in \mathfrak{g}, \alpha_{p, e}(\xi)=0\right\}
$$

and let

$$
\rho_{e}: \mathfrak{g}^{*} \longrightarrow \mathfrak{g}_{e}^{*}
$$

be the transpose of the inclusion map, $\mathfrak{g}_{e} \longrightarrow \mathfrak{g}$. Let $e_{i}, i=1, \ldots, d$ and $e_{i}^{\prime}, i=1, \ldots, d$ be the edges containing $p$ and $q$ respectively, with $e_{d}=e_{d}^{\prime}=e$. Then the $e_{i}$ 's can be ordered so that

$$
\rho_{e} \alpha_{p, e_{i}}=\rho_{e} \alpha_{q, e_{i}^{\prime}}
$$


Proof. (1.18) is just a special case of (1.13), $H$ being the subtorus of $G$ with Lie algebra $\mathfrak{g}_{e}$.

From the data $(\Gamma, \alpha)$ one can construct a graded ring

$$
H(\Gamma, \alpha)=\bigoplus H^{2 k}(\Gamma, \alpha)
$$

as follows. For each edge $e$, the map $\rho_{e}: \mathfrak{g}^{*} \longrightarrow \mathfrak{g}_{e}^{*}$ extends to a ring morphism

$$
\rho_{e}: S\left(\mathfrak{g}^{*}\right) \longrightarrow S\left(\mathfrak{g}_{e}^{*}\right) \text {. }
$$

Let $V_{\Gamma}$ be the set of vertices of $\Gamma$ and let $H^{2 k}(\Gamma, \alpha)$ be the set of all maps

$$
f: V_{\Gamma} \longrightarrow S^{k}\left(\mathfrak{g}^{*}\right)
$$

satisfying the compatibility condition:

$$
\rho_{e} f(p)=\rho_{e} f(q)
$$

for all vertices, $p$ and $q$, and edges, $e$, joining $p$ to $q . H(\Gamma, \alpha)$ can be given a ring structure by pointwise multiplication

$$
\left(f_{1} f_{2}\right)(p)=f_{1}(p) f_{2}(p) .
$$

(Notice that if $f_{1}$ and $f_{2}$ satisfy (1.21) so does $f_{1} f_{2}$ since $\rho_{e}$ is a ring morphism.) In addition, $H(\Gamma, \alpha)$ contains $S\left(\mathfrak{g}^{*}\right)$ as a subring: the ring of constant maps of $V_{\Gamma}$ into $S\left(\mathfrak{g}^{*}\right)$. In particular, $H(\Gamma, \alpha)$ is a module over $S\left(\mathfrak{g}^{*}\right)$.

THEOREM([GKM]). If $M$ is equivariantly formal, $H_{G}(M)$ is isomorphic, as a graded ring, to $H(\Gamma, \alpha)$.

We will sketch a proof of this at the end of $\S 1.8$.

1.7. Holonomy. Let $\mathbb{P}$ be the complex projective line, let $z$ be the standard coordinate function on $\mathbb{C}=\mathbb{P}_{0}=\mathbb{P}-\{\infty\}$ and $w=z^{-1}$ the corresponding coordinate function on $\mathbb{P}_{\infty}=\mathbb{P}-\{0\}$. The multiplicative group, $\mathbb{C}^{\#}=\mathbb{C}-\{0\}$, acts on $\mathbb{C}$ by homotheties:

$$
(a, z) \longrightarrow a z
$$

and this extends to a holomorphic action, $\rho$, of $\mathbb{C}^{\#}$ on $\mathbb{P}$. Let $E$ be a holomorphic, rank $r$ vector bundle over $\mathbb{P}$ and suppose $\rho$ lifts to an action, $\rho$, of $\mathbb{C}^{\#}$ on $E$ by vector bundle automorphisms. Let $E_{0}$ and $E_{\infty}$ be fibers of $E$ over 0 and $\infty$. The isotropy representations of $\mathbb{C}^{\#}$ at 0 and $\infty$ decompose these spaces into invariant one-dimensional subspaces

$$
E_{0}=V_{1} \oplus \cdots \oplus V_{r}
$$

and

$$
E_{\infty}=V_{1}^{\prime} \oplus \cdots \oplus V_{r}^{\prime}
$$

Let $m_{1}, \ldots, m_{r}$ be the weights of the representations of $\mathbb{C}^{\#}$ on $V_{1}, \ldots, V_{r}$. We will assume that these weights are all distinct and that $m_{1}<m_{2}<\cdots<m_{r}$. Similarly 
we will assume that the weights $m_{i}^{\prime}$, of the representations of $\mathbb{C}^{\#}$ on $V_{1}^{\prime}, \ldots, V_{r}^{\prime}$ are all distinct (but we won't require that $m_{1}^{\prime}<\cdots<m_{r}^{\prime}$ ). By a theorem of BirkhoffGrothendieck ([Kl]) there is an equivariant decomposition of $E$ into line-bundles

$$
E=\mathbb{L}_{1} \oplus \cdots \oplus \mathbb{L}_{r}
$$

such that the fiber of $\mathbb{L}_{i}$ over 0 is $V_{i}$. Moreover this decomposition is unique up to isomorphism. (To see this, let

$$
E=\mathbb{L}_{1}^{\prime} \oplus \cdots \oplus \mathbb{L}_{r}^{\prime}
$$

be another decomposition with these properties: Over $\mathbb{P}_{0}$, one can find a trivializing section, $s_{i}$, of $\mathbb{L}_{i}$ which transforms under $\mathbb{C}^{\#}$ according to the law

$$
\rho_{a}^{*} s_{i}=a^{m_{i}} s_{i}
$$

and a trivializing section, $s_{i}^{\prime}$, of $\mathbb{L}_{i}^{\prime}$ with the same transformation property. Moreover, one can assume that $s_{i}(0)=s_{i}^{\prime}(0)$. We claim that $s_{1} \equiv s_{1}^{\prime}$ (and hence $\mathbb{L}_{1}=\mathbb{L}_{1}^{\prime}$ ). To see this we note that because of the transformation law (1.25)

$$
s_{1}^{\prime}=s_{1}+\sum_{i>1} c_{i} z^{m_{1}-m_{i}} s_{i}
$$

on $\mathbb{P}_{0}$. However, since $s_{1}^{\prime}$ is holomorphic near 0 and $m_{1}-m_{i}$ is strictly negative, the constants, $c_{i}$, are all zero.

Applying this argument to the quotient bundle, $E / \mathbb{L}_{1}$, one concludes by induction that $\mathbb{L}_{i} \cong \mathbb{L}_{i}^{\prime}$ for all $i$. Q.E.D.)

In particular, coming back to the isotropy decompositions (1.22) and (1.23), one has a canonical map of the $r$-element set

$$
\left\{V_{1}, \ldots, V_{r}\right\}
$$

onto the $r$-element set

$$
\left\{V_{1}^{\prime}, \ldots, V_{r}^{\prime}\right\}
$$

which maps $V_{i}=\left(\mathbb{L}_{i}\right)_{0}$ onto $\left(\mathbb{L}_{i}\right)_{\infty}$; and we can relabel the $V_{i}^{\prime}$ 's so that this map maps the element, $V_{i}$, of the set (1.26) onto the element, $V_{i}^{\prime}$, of the set (1.27). We will call the map the holonomy map.

Let us apply these remarks to one of the $\mathbb{C} P^{1}$ 's in $\S 1.5$; i.e., let $H$ be a subtorus of $G$ of codimension one and $X=\mathbb{C} P^{1}$ a 2-dimensional connected component of $M^{H}$. Let $N X$ be the normal bundle of $X$ and let $\beta_{1}, \ldots, \beta_{N}$ be the weights of the isotropy representation of $H$ on a typical fiber of $X$. The fiber of $N$ over $x \in X$ splits into a direct sum of vector subspaces

$$
\left(E_{1}\right)_{x} \oplus \cdots \oplus\left(E_{N}\right)_{x}
$$

such that the weight of the representation of $H$ on $\left(E_{i}\right)_{x}$ is $\beta_{i}$. Since the $\beta_{i}$ 's can occur with multiplicities, the dimension of $\left(E_{i}\right)_{x}$ doesn't have to be one; however the assignment, $x \longrightarrow\left(E_{i}\right)_{x}$, defines a complex vector bundle, $E_{i}$, over $X$. Moreover, this bundle can be given a holomorphic structure, and the action of $S^{1}=G / H$ on $E_{i}$ can 
be extended to a holomorphic action of $\mathbb{C}^{\#}$. In particular one can define for $E_{i}$ a holonomy map of the type we described at the beginning of this section.

Now let $X^{G}=\{p, q\}$, and $\alpha_{i, p}$ and $\alpha_{i, q}$ be the weights of the isotropy representations of $G$ on $T_{p} M$ and $T_{q} M$. By assumption these weights occur with multiplicity one, so one gets decompositions of $T_{p} M$ and $T_{q} M$ into direct sums of one-dimensional weight spaces

$$
T_{p}^{\alpha_{1, p}} \oplus \cdots \oplus T_{p}^{\alpha_{d, p}}
$$

and

$$
T_{q}^{\alpha_{1, q}} \oplus \cdots \oplus T_{q}^{\alpha_{d, q}} .
$$

Let $\rho$ be the projection of $\mathfrak{g}^{*}$ onto $\mathfrak{h}^{*}$. By (1.18) we can reorder the $\alpha_{i, q}$ 's so that $\rho\left(\alpha_{i, p}\right)=\beta_{i}=\rho\left(\alpha_{i, q}\right)$; so, if the $\beta_{i}$ 's are all distinct, one has a canonical map of the set

$$
\left\{\alpha_{1, p}, \ldots, \alpha_{d, p}\right\}
$$

onto the set

$$
\left\{\alpha_{1, q}, \ldots, \alpha_{d, q}\right\}
$$

mapping $\alpha_{i, p}$ onto $\alpha_{i, q}$. However, this canonical map can even be defined when the $\beta_{i}$ 's are not distinct (that is, when the vector bundles, $E_{i}$, are not of rank one) by using the holonomy structure on these bundles. In other words there exists a canonical holonomy mapping from the set (1.29) to the set (1.30) which, when the $\beta_{i}$ 's are distinct, is defined trivially by the recipe

$$
\alpha_{i, p} \longrightarrow \rho\left(\alpha_{i, p}\right)=\beta_{i}=\rho\left(\alpha_{i, q}\right) \longrightarrow \alpha_{i, q},
$$

but, when the $\beta_{i}$ 's are not distinct, involves some topological properties of the bundles, $E_{i}$. We will denote this map by $\theta_{p, e}$.

One can give a slightly more "graphical" description of this map: As in $\S 1.6$, let $\Gamma$ be the GKM graph, let $V_{\Gamma}$ be its vertices and let $I_{\Gamma}$ be the incidence relation. The projection, $I_{\Gamma} \longrightarrow V_{\Gamma}$, can be regarded as a fiber bundle over $V_{\Gamma}$, the fiber, $E_{p}$, over $p \in V_{\Gamma}$ being the set of all pairs, $(p, e)$ in $I_{\Gamma}$ (i.e., the set of all oriented edges of $\Gamma$ pointing out from $p$ ). In this "fiber bundle" picture, $\theta_{p, e}$ is just a map

$$
\theta_{p, e}: E_{p} \longrightarrow E_{q}
$$

with the properties

$$
\theta_{p, e}(p, e)=(q, e)
$$

and

$$
\theta_{p, e}=\theta_{q, e}^{-1}
$$

$e$ being the edge joining $p$ to $q$. A family of maps, $\theta$, with the properties (1.32)-(1.33) is called a connection on $\Gamma$ (cf. [CS]). In terms of this connection, one can reformulate (1.18) more precisely:

THEOREM 1.1. Let $e$ be an edge of $\Gamma$ joining the vertex, $p$, to the vertex, $q$. Then for every $\left(p, e_{i}\right) \in E_{p}$

$$
\rho_{e}\left(\alpha_{p, e_{i}}\right)=\rho_{e}\left(\alpha_{q, e_{i}^{\prime}}\right),
$$

$\left(q, e_{i}^{\prime}\right)$ being the image with respect to $\theta_{p, e}$ of $\left(p, e_{i}\right)$. 
1.8. Betti numbers. The theorem of Goresky-Kottwitz-MacPherson described in $\S 1.6$ implies that the odd Betti numbers of $M$ are zero. The even Betti numbers can be computed as follows. As in $\S 1.3$ let $K$ be a one-dimensional closed connected subgroup of $G$ with $M^{G}=M^{K}$ and let $\xi$ be a basis vector of $\mathfrak{k}$. For every $p \in V_{\Gamma}$ let $\sigma_{p}$ be the number of edges, $e$, with one vertex $p$ and with $\alpha_{p, e}(\xi)<0$.

ThEOREM. The $2 k$-th Betti number, $\beta_{k}$, of $M$ is equal to the number of points, $p \in V_{\Gamma}$, with $\sigma_{p}=k$.

If $M$ possesses a $G$-invariant symplectic form having the properties described in $\S 1.3$, this theorem can be proved by Morse theory : Let $f$ be the function defined by (1.9). The critical points of this function coincide with the fixed points of $G$ and it is not difficult to show that the index of the Hessian of $f$ at $p \in M^{G}$ is just $2 \sigma_{p}$.

We will now sketch a proof of the Goresky-Kottwitz-MacPherson theorem. It is clear that

$$
H_{G}\left(M^{G}\right)=H^{0}\left(M^{G}\right) \otimes S\left(\mathfrak{g}^{*}\right)=\operatorname{Maps}\left(V_{\Gamma}, S\left(\mathfrak{g}^{*}\right)\right) ;
$$

so if $i: M^{G} \longrightarrow M$ is the inclusion, there is an induced map

$$
i^{*}: H_{G}(M) \longrightarrow \operatorname{Maps}\left(V_{\Gamma}, S\left(\mathfrak{g}^{*}\right)\right) \text {. }
$$

It is easy to see that the image of $i^{*}$ is contained in $H(\Gamma, \alpha)$. Moreover, by a classical theorem of Borel, $i^{*}$ is an isomorphism modulo torsion; in particular, the kernel of $i^{*}$ is a torsion submodule of $H_{G}(M)$. However, by (1.6), $H_{G}(M)$ is a free $S\left(\mathfrak{g}^{*}\right)$-module; so $i^{*}: H_{G}^{2 k}(M) \longrightarrow H^{2 k}(\Gamma, \alpha)$ is injective and

$$
\operatorname{dim} H_{G}^{2 k}(M)=\sum \beta_{r} \operatorname{dim} S^{k-r}\left(\mathfrak{g}^{*}\right) .
$$

Therefore, in order to prove that

$$
\operatorname{dim} H^{2 k}(\Gamma, \alpha)=\sum \beta_{r} \operatorname{dim} S^{k-r}\left(\mathfrak{g}^{*}\right),
$$

it suffices to show that the dimension of $H^{2 k}(\Gamma, \alpha)$ is less than or equal to the right hand side of (1.35), and we will prove this (by a very simple algebraic argument) in section 2.9 .

2.1. Graphs and axial functions. In part 2 of this article, $\mathfrak{g}$ will simply be a vector space over $\mathbb{R}$ of dimension $n$ and $\mathfrak{g}^{*}$ its vector space dual. (In particular $\mathfrak{g}$ will not necessarily be the Lie algebra of a group, $G$.) Let $\Gamma$ be a finite simple $d$-valent graph and $I_{\Gamma}$ its incidence relation.

Definition. 1. An axial function on $\Gamma$ is a map $\alpha: I_{\Gamma} \longrightarrow \mathfrak{g}^{*}-\{0\}$ having the properties (1.16)-(1.18).

Note that by axiom (1.16) an axial function can be thought as a function

$$
\alpha: E_{\Gamma}^{ \pm} \longrightarrow \mathfrak{g}^{*}
$$

on the set of oriented edges of $\Gamma$, having the property that if $e^{+}$and $e^{-}$are the two oriented edges of $\Gamma$ associated with an unoriented edge $e$, then

$$
\alpha\left(e^{+}\right)=-\alpha\left(e^{-}\right)
$$


Definition. A connection, $\theta$, on $\Gamma$ is a collection of maps, $\theta_{p, e},(p, e) \in I_{\Gamma}$, satisfying the axioms (1.31)-(1.33); an axial function, $\alpha$, is compatible with $\theta$ if it satisfies the stronger version, (1.34) of axiom (1.18).

For instance, suppose that for every vertex, $p$, and every edge, $e$, containing $p$, no two of the vectors (1.18) are equal. Then, as we showed in section 1.7, there is a unique connection on $\Gamma$ which is compatible with $\alpha$.

We will list below a few examples of axial functions and connections on graphs and describe some of their functorial properties:

EXAMPLE 1. The complete graph on $N$ vertices. The vertices of this graph are the elements of the $N$-element set $\{1, \ldots, N\}$ and each pair of elements, $(i, j), i \neq j$, is joined by an edge. Thus the set of oriented edges is just the set

$$
\{(i, j) ; 1 \leq i, j \leq N, i \neq j\} \text {. }
$$

Let $\alpha_{i}, i=1, \ldots, N$ be non-zero elements of $\mathfrak{g}^{*}$. Then the function $(i, j) \longrightarrow \alpha_{i}-\alpha_{j}$ satisfies (1.16) and (1.18) and hence it is an axial function iff, for every $i$, the $N-1$ vectors $\alpha_{i}-\alpha_{j}, i \neq j$ are pairwise linearly independent. Conversely, we claim that every axial function is of this form. (Proof : Let $(i, j) \longrightarrow \alpha_{i, j}$ be an axial function. Then if $i, j$ and $k$ are distinct

$$
\alpha_{i, k}=\alpha_{j, k}+c_{i, j} \alpha_{i, j} \quad c_{i, j}=c_{j, i} .
$$

Hence

$$
\alpha_{k, i}=\alpha_{j, i}+c_{k, j} \alpha_{k, j}=\alpha_{k, j}+c_{i, j} \alpha_{j, i}
$$

so $\left(1-c_{i, j}\right) \alpha_{j, i}=\left(1-c_{k, j}\right) \alpha_{k, j}$ and $c_{i, j}=c_{k, j}=1$. Now let $\alpha_{1}=0$ and let $\alpha_{i}=\alpha_{i, 1}$ for $i>1$.)

An $\alpha$-compatible connection, $\theta$, can be defined as follows: For every oriented edge, $(i, j)$, of $\Gamma$ let $\theta_{i, j}$ be the map of the set of edges of $\Gamma$ containing $i$ onto the set of edges of $\Gamma$ containing $j$ which maps $(i, j)$ onto $(j, i)$ and, for $k \neq i, j$, maps $(i, k)$ onto $(j, k)$.

EXAMPLE 2. Sub-objects. Let $\Gamma_{1}$ be an $r$-valent sub-graph of $\Gamma$ and $j$ the embedding of $V_{\Gamma_{1}}$ into $V_{\Gamma}$. From $j$ one gets an embedding $i: I_{\Gamma_{1}} \longrightarrow I_{\Gamma}$ and one can pull-back the axial function $\alpha$ to $I_{\Gamma_{1}}$. In general $i^{*} \alpha$ won't be an axial function; however if it is, we will say that $\Gamma_{1}$ is compatible with $\alpha$ and call $\left(\Gamma_{1}, i^{*} \alpha\right)$ a sub-object of $(\Gamma, \alpha)$.

EXAMPLE 3. Sub-objects which are "totally geodesic" with respect to a connection. Let $\theta$ be a connection on $\Gamma$, and let $\Gamma^{\prime}$ be an $r$-valent subgraph of $\Gamma$. For every vertex, $p$, of $\Gamma$ let $E_{p}$ and $E_{p}^{\prime}$ be the oriented edges of $\Gamma$ and of $\Gamma^{\prime}$ issuing from $p$. We will say that $\Gamma^{\prime}$ is totally geodesic with respect to $\theta$ if, for every oriented edge, $(p, q)$, of $\Gamma^{\prime}$ the restriction of the holonomy map, $\theta_{p, q}: E_{p} \longrightarrow E_{q}$, to $E_{p}^{\prime}$ maps $E_{p}^{\prime}$ onto $E_{q}^{\prime}$.

If this happens, this restriction defines an induced connection, $\theta^{\prime}$, on $\Gamma^{\prime}$. Moreover, if $\alpha: I_{\Gamma} \longrightarrow \mathfrak{g}^{*}$ is an axial function which is compatible with $\theta$, the restriction of $\alpha$ to $I_{\Gamma^{\prime}}$ is an axial function and is compatible with $\theta^{\prime}$.

EXAMPLE 4. The totally geodesic sub-objects of $\Gamma_{N}$. Let $\Gamma_{N}$ be the complete graph on $N$ vertices and for every subset, $S$, of $\{1, \ldots, N\}$ let $\Gamma_{S}$ be the graph 
whose vertices are the elements of $S$ and whose oriented edges are the pairs $\left(s_{1}, s_{2}\right)$, $\left(s_{i} \in S\right), s_{1} \neq s_{2}$. It is obvious that $\Gamma_{S}$ is a totally geodesic with respect to the connection we defined in example 1 ; and, in fact, every totally geodesic sub-object of $\Gamma_{N}$ is a $\Gamma_{S}$ for some subset $S$. (To see this, let $\Gamma^{\prime}$ be a connected totally geodesic subgraph and let $\left(p_{1}, p_{2}\right)$ be an oriented edge of $\Gamma^{\prime}$. If $q$ is a vertex of $\Gamma^{\prime}$ distinct from $p_{1}$ and $p_{2}$ and $\left(p_{1}, q\right)$ is an oriented edge of $\Gamma^{\prime},\left(p_{2}, q\right)$ has to be an oriented edge of $\Gamma^{\prime}$,so it follows from the connectivity of $\Gamma^{\prime}$ that, for every pair of vertices, $p$ and $q$, of $\Gamma^{\prime}(p, q)$ is an oriented edge of $\left.\Gamma^{\prime}.\right)$

EXAMPLE 5. The graph $\Gamma_{\mathfrak{h}}$. Let $\mathfrak{h}$ a vector subspace of $\mathfrak{g}$ and let $\rho_{\mathfrak{h}}: \mathfrak{g}^{*} \longrightarrow \mathfrak{h}^{*}$ be the transpose of the inclusion map $\mathfrak{h} \longrightarrow \mathfrak{g}$. Let $\Gamma_{\mathfrak{h}}$ be the subgraph of $\Gamma$ whose edges are the edges, $e$, of $\Gamma$ for which

$$
\rho_{\mathfrak{h}} \alpha_{p, e}=-\rho_{\mathfrak{h}} \alpha_{q, e}=0,
$$

$p$ and $q$ being the vertices of $e$. Each connected component of this graph is $k$-valent for some $k$ and is a sub-object in the sense of item 2. Moreover, if $p$ and $q$ are in the same connected component of $\Gamma_{\mathfrak{h}}$ and $e_{i}$ and $e_{i}^{\prime}, i=1, \ldots, d$, are the edges of $\Gamma$ containing $p$ and $q$, one can order the $e_{i}$ 's so that

$$
\rho_{\mathfrak{h}} \alpha_{p, e_{i}}=\rho_{\mathfrak{h}} \alpha_{q, e_{i}^{\prime}}
$$

(compare with (1.13)).

EXAmple 6. Product objects. Let $\Gamma_{1}$ be a graph of valence $d_{1}$ and $\Gamma_{2}$ a graph of valence $d_{2}$. The vertices of the product graph, $\Gamma_{1} \times \Gamma_{2}$, are the pairs $(p, q), p \in V_{\Gamma_{1}}$ and $q \in V_{\Gamma_{2}}$; two vertices $(p, q)$ and $\left(p^{\prime}, q^{\prime}\right)$ are joined by an edge if either $p=p^{\prime}$ and $q$ and $q^{\prime}$ are joined by an edge in $\Gamma_{2}$ or $q=q^{\prime}$ and $p$ and $p^{\prime}$ are joined by an edge in $\Gamma_{1}$. Thus this product graph is a $d_{1}+d_{2}$-valent graph and its set of oriented edges is the disjoint union

$$
E_{\Gamma_{1}}^{ \pm} \amalg E_{\Gamma_{2}}^{ \pm}
$$

of the set of oriented edges of $\Gamma_{1}$ and the set of oriented edges of $\Gamma_{2}$. If

$$
\alpha_{i}: E_{\Gamma_{i}}^{ \pm} \longrightarrow \mathfrak{g}^{*}, \quad i=1,2
$$

is an axial function on $\Gamma_{i}$, one defines the product function $\alpha$ on $\Gamma_{1} \times \Gamma_{2}$ to be the function which is equal to $\alpha_{1}$ on the first summand of (2.3) and equal to $\alpha_{2}$ on the second summand. Then $\alpha$ satisfies (1.16) - (1.18) and it is called the product axial function. If in addition, $\theta_{1}$ and $\theta_{2}$ are connections on $\Gamma_{1}$ and $\Gamma_{2}$ one can define a product connection, $\theta$, on $\Gamma_{1} \times \Gamma_{2}$ by letting

$$
\theta_{\left(p, q ; p^{\prime}, q\right)}=\theta_{p, p^{\prime}} \times(\text { Identity })_{q, q}
$$

and letting

$$
\theta_{\left(p, q ; p, q^{\prime}\right)}=(\text { Identity })_{p, p} \times \theta_{q, q^{\prime}}
$$

If $\alpha_{i}$ and $\theta_{i}$ are compatible, for $i=1$ and 2, the product axial function which we defined above is compatible with $\theta$. 
EXAMPLE 7. Blowing-up. This operation can be defined for any sub-object of a graph-axial function pair; however, for simplicity, we will only consider here the special case when the sub-object is a point. Let $\Gamma$ be a finite simple $d$-valent graph and let $p_{0}$ be an arbitrary vertex of $\Gamma$. Let $e_{i}, 1=1, \ldots, d$ be the edges of $\Gamma$ containing $p_{0}$ and let $q_{i}$ be the vertex joined by $e_{i}$ to $p_{0}$. From this data one can construct a new graph, $\Gamma^{\#}$, as follows. Replace the vertex $p_{0}$ by $d$ new vertices, $p_{i}, i=1, \ldots, d$ ( which one should think of as being the "baricenters" of the edges $e_{i}$ ) and to each of these new vertices adjoin $d$ edges; one edge going from $p_{i}$ to $q_{i}$ (which one should think of as being a replacement for the old edge $e_{i}$ ) and one edge going from $p_{i}$ to each of the $p_{j}^{\prime} s, j \neq i$. Let

$$
\beta: V_{\Gamma \#} \longrightarrow V_{\Gamma}
$$

be the map which sends $\left\{p_{1}, \ldots, p_{d}\right\}$ to $p_{0}$ and is the identity on the complement of $\left\{p_{1}, \ldots, p_{d}\right\}$. We will call this map the blowing-down map. The set $\left\{p_{1}, \ldots, p_{d}\right\}$, which is the pre-image of $p_{0}$ with respect to $\beta$, is the set of vertices of a sub-graph, $\Gamma_{0}$, of $\Gamma^{\#}$ (the complete graph on $d$ vertices) which we will call the singular locus of the blowing-down map $\beta$.

Now let $\alpha: I_{\Gamma} \longrightarrow \mathfrak{g}^{*}$ be an axial function and let $\alpha_{i}, i=1, \ldots, d$ be the values of $\alpha$ on the vertex-edge pairs $\left(p_{0}, e_{i}\right)$. Let us assume that for each $i$ the $d-1$ vectors $\alpha_{j}-\alpha_{i}, j \neq i$ are pairwise linearly independent. We can then define an axial function, $\alpha^{\#}$, on $\Gamma^{\#}$, as follows:

1. On the oriented edges, $e$, of $\Gamma$, not containing $p_{0}, \alpha^{\#}(e)=\alpha(e)$.

2. On the oriented edges, $e=\left(p_{i}, q_{i}\right), \alpha^{\#}(e)=\alpha_{i}$.

3. On the oriented edges, $e=\left(p_{i}, p_{j}\right), \alpha^{\#}(e)=\alpha_{j}-\alpha_{i}$.

This defines $\alpha^{\#}$ on all edges of $\Gamma^{\#}$ and it is easy to check that $\alpha^{\#}$ satisfies the axioms (1.16) - (1.18).

Finally, if $\theta$ is a connection on $\Gamma$ there is a unique connection, $\theta^{\#}$, on $\Gamma^{\#}$ with the following properties.

1. The restriction of $\theta$ to $\Gamma_{0}$ is the connection described in example one.

2. $\theta_{\left(p_{i}, p_{j}\right)}^{\#}$ maps the oriented edge, $\left(p_{i}, q_{i}\right)$, to the oriented edge $\left(p_{j}, q_{j}\right)$.

3. $\theta_{\left(p_{i}, q_{i}\right)}^{\#}$ maps the oriented edge $\left(p_{i}, q_{i}\right)$ to the oriented edge $\theta_{\left(p, q_{i}\right)}\left(e_{j}\right)$.

4. If $q$ is not equal to one of the $p_{i}$ 's and $q$ and $q_{i}$ are joined by an edge,

$$
\theta_{\left(q_{i}, q\right)}^{\#}\left(e_{i}^{\#}\right)=\theta_{\left(q_{i}, q\right)}\left(e_{i}^{-}\right)
$$

$e_{i}^{\#}$ being the edge joining $q_{i}$ to $p_{i}$ and $e_{i}^{-}$the edge joining $q_{i}$ to $p$. On the other edges of $\Gamma^{\#}$ issuing from $q_{i}, \theta_{\left(q_{i}, q\right)}^{\#}=\theta_{\left(q_{i}, q\right)}$.

5. If $q$ and $q^{\prime}$ are not equal to one of the $q_{i}$ 's or one of the $p_{i}{ }^{\prime} \mathrm{s}, \theta_{\left(q, q^{\prime}\right)}^{\#}=\theta_{\left(q, q^{\prime}\right)}$.

EXAMPLE 8. The case $d=d i m \mathfrak{g}^{*}=2$. Let $\Gamma$ be a finite connected 2-valent graph with $N$ vertices, $\mathfrak{g}^{*}$ a 2 -dimensional vector space and $\alpha$ an axial function. We will orient the edges of $\Gamma$ so that for each vertex, $p$, one of the edges containing $p$ is pointing in the direction of $p$ and the other is pointing away from $p$ (since $\Gamma$ is connected there are clearly just two ways of orienting the edges so that their orientations have this property.). Let $p_{1}, \ldots, p_{N}, p_{N+1}=p_{1}$ be an enumeration of the vertices of $\Gamma$ such that the outward pointing edge at $p_{i}$ joins $p_{i}$ to $p_{i+1}$ and let $\alpha_{i}$ be the value of $\alpha$ on 
$\left(p_{i}, p_{i+1}\right)$. Then $(1.18)$ is equivalent to

$$
\alpha_{i+1} \wedge \alpha_{i}=\alpha_{i} \wedge \alpha_{i-1}
$$

for all $i$. ( For example for $N=4 k$, let $\left\{\alpha_{1}, \alpha_{2}\right\}$ be a basis of $\mathfrak{g}^{*}$. Then a solution of (2.5) is obtained by letting $\alpha_{1}=-\alpha_{3}=\alpha_{5}=\ldots$ and $\alpha_{2}=-\alpha_{4}=\alpha_{6}=\ldots$ ).

2.2. Orientations. Let $(\Gamma, \alpha)$ be a graph-axial function pair and let

$$
\mathcal{P}=\left\{\xi \in \mathfrak{g}, \alpha_{p, e}(\xi) \neq 0 \text { for all }(p, e) \in I_{\Gamma}\right\}
$$

Then for every $\xi \in \mathcal{P}$, the axial function $\alpha$ defines an orientation of $\Gamma$; in other words, for each edge $e$, it fixes an ordering of the vertices of $e$. Namely if $p$ and $q$ are the vertices of $e$, one orders them so that

$$
p<q \Leftrightarrow \alpha_{p, e}(\xi)>0 .
$$

It is clear that this orientation doesn't depend on $\xi$ but only on the connected component of $\mathcal{P}$ in which $\xi$ is contained. On the other hand it is clear that different components will give rise to different orientations (for instance, replacing $\xi$ by $-\xi$ reverses all the orientations). We will say that $\Gamma$ satisfies the no-cycle condition if, for at least one of these orientations, $\Gamma$ has no cycles.

Definition. Given $\xi \in \mathcal{P}$, a function $f: V_{\Gamma} \longrightarrow \mathbb{R}$ is positively oriented with respect to $\xi$ if, for every pair of vertices, $p$ and $q$, and edge, $e$, joining $p$ to $q$, the ratio of $f(p)-f(q)$ to $\alpha_{q, e}(\xi)$ is positive.

If $f$ is positively oriented with respect to $\xi$, the orientation of $\Gamma$ associated with $\xi$ can't have closed cycles since $f$ has to be strictly increasing along any oriented path. We will prove that the converse is true:

THEOREM. If the orientation of $\Gamma$ associated with $\xi$ has no cycles, there exists a function $f: V_{\Gamma} \longrightarrow \mathbb{R}$ which is positively oriented with respect to $\xi$.

Proof. Given $p \in V_{\Gamma}$, consider the longest oriented path with initial point $p$, i.e., the longest sequence

$$
p=p_{1}, p_{2}, \ldots, \quad p_{i} \in V_{\Gamma}
$$

with the property that $p_{i}$ and $p_{i+1}$ are the vertices of a common edge and, relative to the orientation on this edge, $p_{i}<p_{i+1}$. If $\Gamma$ has no cycles this longest path has to be of finite length, i.e., has to terminate at some point, $p_{N}$. Now set $f(p)=-N$. It is easy to check that this function is positively oriented with respect to $\xi$.

\section{REMARKS.}

1. The vertices, $p$, where $f(p)=0$ have the property that all edges containing $p$ are pointing "into" $p$, i.e. $p$ is a "maximum" of the oriented graph $\Gamma$. In particular, if $f(p)=-N$, this is true of the vertex $p_{N}$ in the sequence (2.7); so the argument above shows that every vertex can be joined by an oriented path to a maximal vertex. 
2. One can perturb $f$ so that it remains positively oriented with respect to $\xi$ and, in addition, takes on distinct values at distinct vertices. Namely suppose that

$$
f^{-1}(k)=\left\{p_{1}, \ldots, p_{r}\right\} .
$$

Redefine $f$ on the set $\left\{p_{1}, \ldots, p_{r}\right\}$ by setting $f$ equal to $k+\epsilon_{i}$ on $p_{i}$ where $\epsilon_{i} \neq$ $\epsilon_{j}$ for $i \neq j$ and the $\epsilon_{i}$ 's are small. This redefined function is still positively oriented with respect to $\xi$ but now takes distinct values at $p_{1}, \ldots, p_{r}$.

2.3. The cohomology ring of $(\Gamma, \alpha)$. We define the cohomology ring of $(\Gamma, \alpha)$ to be the ring $H(\Gamma, \alpha)$ which we defined in $\S 1.6$. As $(\Gamma, \alpha)$ is no longer the GKM data associated with a $G$ - manifold, it is, perhaps, a misnomer to refer to this ring as a "cohomology ring"; however, there are other reasons for using this terminology. For instance, if $\Gamma$ is the one-skeleton of a simplicial polytope, $H(\Gamma, \alpha)$ is just the StanleyReissner cohomology ring of the dual polytope. (We are indebted to Mark Goresky for this observation.) We will describe below a few properties of this ring.

1. As we pointed out in $\S 1.6, H(\Gamma, \alpha)$ contains $S\left(\mathfrak{g}^{*}\right)$ as a subring.

2. Chern classes: For each $p \in V_{\Gamma}$, let $e_{1}, \ldots, e_{d}$ be the edges containing $p$ and let $c_{k}(p)$ be the $k$-th elementary symmetric function in the monomials $\alpha_{p, e_{1}}, \ldots, \alpha_{p, e_{d}}$. The function $p \longrightarrow c_{k}(p)$ defines an element $c_{k}$ of $H^{2 k}(\Gamma, \alpha)$ which can be thought of as the $k$-th Chern class of the "tangent bundle" of $\Gamma$.

3. Symplectic structures: An element of $H^{2}(\Gamma, \alpha)$ is just a map $c: V_{\Gamma} \longrightarrow \mathfrak{g}^{*}$ satisfying

$$
c(p)-c(q)=\lambda_{e} \alpha_{q, e}
$$

for every pair of vertices, $p, q$, and edge, $e$, joining $p$ to $q$. We will call $c$ symplectic if, for every edge $e, \lambda_{e}$ is positive. The existence of a symplectic structure implies that for every $\xi \in \mathcal{P}$, the orientation of $\Gamma$ associated with $\xi$ has the no-cycle property. (Proof: It follows from (2.8) that the $\xi$-component of $c$ is an $\mathbb{R}$-valued function on $V_{\Gamma}$ which is positively oriented with respect to $\xi$.)

4. Thom classes: Fix a vertex $p$ and let $e_{1}, \ldots, e_{d}$ be the edges containing $p$. Let $\tau: V_{\Gamma} \longrightarrow S^{d}\left(\mathfrak{g}^{*}\right)$ be the map which is zero at $q \neq p$ and at $p$ is equal to $\alpha_{p, e_{1}} \ldots \alpha_{p, e_{d}}$. Then $\tau$ is in $H^{2 d}(\Gamma, \alpha)$.

5. Sub-objects: Let $\Gamma_{1}$ be a sub-graph of $\Gamma$ which is compatible with $\alpha$. Then the inclusion map $j: V_{\Gamma_{1}} \longrightarrow V_{\Gamma}$ induces a map

$$
j^{*}: H(\Gamma, \alpha) \longrightarrow H\left(\Gamma_{1}, \alpha_{1}\right), \quad \alpha_{1}=i^{*} \alpha .
$$

6. Gysin maps: Suppose that $\Gamma_{1}$ is compatible with $\alpha$. The Thom class of $\Gamma_{1}$ is the map $\tau: V_{\Gamma} \longrightarrow S^{d-r}\left(\mathfrak{g}^{*}\right)$ which is zero on the vertices of $\Gamma$ which are not vertices of $\Gamma_{1}$ and on vertices, $p$, of $\Gamma_{1}$, is equal to

$$
\tau(p)=\alpha_{p, e_{1}} \ldots \alpha_{p, e_{s}}
$$

where $s=d-r$ and $e_{1}, \ldots, e_{s}$ are the edges of $\Gamma$ at $p$ which don't belong to $\Gamma_{1}$.

From $\tau$ one gets a Gysin map

$$
H^{2 k}\left(\Gamma_{1}, \alpha_{1}\right) \longrightarrow H^{2(k+s)}(\Gamma, \alpha)
$$

mapping $f$ to $\tau f$. (Since $\tau$ is supported on $V_{\Gamma}$, this map is well-defined.) 
7. The cohomology of blow-ups: Let $\Gamma$ be a $d$-valent graph and $\alpha: I_{\Gamma} \longrightarrow \mathfrak{g}^{*}$ an axial function. Let $p_{0}$ be a vertex of $\Gamma$ and $\left(\Gamma^{\#}, \alpha^{\#}\right)$ the blow-up of $(\Gamma, \alpha)$ at $p_{0}$ (See $\S 2.1$ ). From the blowing down map (2.4) one gets a pull-back map on cohomology

$$
\beta^{*}: H(\Gamma, \alpha) \longrightarrow H\left(\Gamma^{\#}, \alpha^{\#}\right)
$$

which embeds $H(\Gamma, \alpha)$ as a subring of $H\left(\Gamma^{\#}, \alpha^{\#}\right)$. Moreover the singular locus, $\Gamma_{0}$, of $\beta$, is a sub-object of $\Gamma^{\#}$ (in the sense of example 2 above) and its Thom class,

$$
\tau \in H^{2}\left(\Gamma^{\#}, \alpha^{\#}\right)
$$

generates $H\left(\Gamma^{\#}, \alpha^{\#}\right)$ over the sub-ring $H(\Gamma, \alpha)$, subject to the relation

$$
\tau^{d}-c_{1} \tau^{d-1}+c_{2} \tau^{d-2}-\ldots \pm c_{d}
$$

the $c_{i}$ 's being the Chern classes of $\Gamma$ for $i<d$ and $c_{d}$ being the Thom class of $p_{0}$.

2.4. The Atiyah-Bott-Berline-Vergne localization theorem. We have just discussed functoriality for sub-objects of $\Gamma$. What about quotient objects? To take the most extreme case let " $p t$ " be the trivial zero-valent graph consisting of one vertex, $p t$, and no edges and let

$$
\pi: V_{\Gamma} \longrightarrow p t
$$

be the constant map. We have already seen (see $\S 2.3$ item 1 ) that there is a functorial map

$$
\pi^{*}: H^{2 k}(p t) \longrightarrow H^{2 k}(\Gamma, \alpha) .
$$

However, does there exist a Gysin map

$$
\pi_{*}: H^{2 k}(\Gamma, \alpha) \longrightarrow H^{2(k-d)}(p t) ?
$$

- Such a map, if it existed, would have to have the following property. Let $p$ be a vertex of $\Gamma$ and let $j_{p}: p t \longrightarrow V_{\Gamma}$ be the map $p t \longrightarrow p$. Then, by functoriality, $\pi_{*}$ would have to satisfy

$$
\pi_{*}\left(j_{p}\right)_{*}=\text { identity }
$$

and, by items 4 and 6 of $\S 2.3, \pi_{*}$ would have to have the form

$$
\pi_{*} f=\sum \frac{f(p)}{\prod \alpha_{p, e}} .
$$

However, it is by no means obvious that this map is well defined, i.e. that the right hand side of $(2.9)$ is in $S\left(g^{*}\right)$. We will prove that it is :

Theorem 2.2. $\pi_{*}$ maps $H^{2 k}(\Gamma, \alpha)$ into $S^{k-d}\left(\mathfrak{g}^{*}\right)$.

Proof. Let $f \in H^{2 k}(\Gamma, \alpha)$; then $\pi_{*} f$ can be written as

$$
\pi_{*} f=\frac{g}{\prod_{j=1}^{N} \alpha_{j}}
$$


where $g \in S^{k-d+N}\left(\mathfrak{g}^{*}\right)$ and $\alpha_{1}, \cdots, \alpha_{N}$ are pair-wise linearly independent. We will show that $\alpha_{1}$ divides $g$.

The vertices of $\Gamma$ can be divided into two categories:

1. The first subset, $V_{1}$, contains the vertices $p \in V_{\Gamma}$ for which none of the $\alpha_{p, e}$ 's is a multiple of $\alpha_{1}$

2. The second subset, $V_{2}$, contains the vertices $p \in V_{\Gamma}$ for which there exists an edge $e$ such that $\alpha_{p, e}$ is a multiple of $\alpha_{1}$. ( Notice that 1.17 implies that there will be exactly one such edge. )

The part of (2.9) corresponding to vertices in the first category will then be of the form

$$
\sum_{p \in V_{1}} \frac{f(p)}{\prod \alpha_{p, e}}=\frac{g_{1}}{\prod_{j=2}^{N} \alpha_{j}}
$$

with $g_{1} \in S\left(\mathfrak{g}^{*}\right)$.

If $p \in V_{2}$ then there exists an edge $e$ from $p$ such that $\alpha_{p, e}=\lambda \alpha_{1}$ with $\lambda \in \mathbb{C}-\{0\}$; let $q$ be the other vertex of $e$. Since $\alpha_{q, e}=-\alpha_{p, e}$ it results that $q \in V_{2}$ as well and thus the vertices in $V_{2}$ can be paired as above.

Let $e_{i}, i=1, \ldots, d$ and $e_{i}^{\prime}, i=1, \ldots, d$ be the edges containing $p$ and $q$ respectively, with $e_{d}=e_{d}^{\prime}=e$. Then the $e_{i}$ 's can be ordered (cf. 1.18) so that

$$
\alpha_{p, e_{i}} \equiv \alpha_{q, e_{i}^{\prime}} \quad\left(\bmod \alpha_{1}\right)
$$

Also (1.21) implies that

$$
f(q) \equiv f(p) \quad\left(\bmod \alpha_{1}\right)
$$

The part of (2.9) corresponding to $p$ and $q$ is given by

$$
\frac{f(p)}{\prod_{j=1}^{d} \alpha_{p, e_{j}}}+\frac{f(q)}{\prod_{j=1}^{d} \alpha_{q, e_{j}^{\prime}}}=\frac{f(p) \alpha_{q, e_{1}^{\prime}} . . \alpha_{q, e_{d-1}^{\prime}}-f(q) \alpha_{p, e_{1}} . . \alpha_{p, e_{d-1}}}{\lambda \alpha_{1} \alpha_{p, e_{1}} \ldots \alpha_{q, e_{d-1}^{\prime}}} .
$$

But multiplying together the congruences (2.12) and (2.13) we obtain that $\alpha_{1}$ divides the numerator of $(2.10)$ so that

$$
\frac{f(p)}{\prod_{j=1}^{d} \alpha_{p, e_{j}}}+\frac{f(q)}{\prod_{j=1}^{d} \alpha_{q, e_{j}^{\prime}}}=\frac{g_{p, q}}{\prod_{j=2}^{N} \alpha_{j}}
$$

with $g_{p, q} \in S\left(\mathfrak{g}^{*}\right)$. Therefore

$$
\sum_{p \in V_{2}} \frac{f(p)}{\prod \alpha_{p, e}}=\frac{g_{2}}{\prod_{j=2}^{N} \alpha_{j}}
$$

with $g_{2} \in S\left(\mathfrak{g}^{*}\right)$. Adding (2.11) and (2.16) we get that

$$
\frac{g}{\prod_{j=1}^{N} \alpha_{j}}=\frac{g_{1}+g_{2}}{\prod_{j=2}^{N} \alpha_{j}}
$$

with $g_{1}+g_{2} \in S\left(\mathrm{~g}^{*}\right)$, i.e. that $\alpha_{1}$ divides $g$. The same argument can be used to show that each $\alpha_{j}$ divides $g$ and therefore $\pi_{*} f \in S^{k-d}\left(\mathfrak{g}^{*}\right)$, as desired. 
2.5. The Kirwan map. From now on we will assume that $(\Gamma, \alpha)$ satisfies the no-cycle condition. Let $\xi$ be an element of $\mathcal{P}$ which gives an orientation of $\Gamma$ without cycles and let $\phi: V_{\Gamma} \longrightarrow \mathbb{R}$ be positively oriented with respect to $\xi$; without loss of generality we can assume that $\phi$ is injective. For $c \in \mathbb{R}-\phi\left(V_{\Gamma}\right)$, we define the $c$-cross section, $\Gamma_{c}$, of $\Gamma$ to be the set of edges, $e$, of $\Gamma$ with the property that, for one of the vertices, $p$, of $e, \phi(p)>c$ and for the other vertex, $q, \phi(q)<c$. Let $\mathfrak{g}_{\xi}^{*}$ be the annihilator of $\xi$ in $\mathfrak{g}^{*}$ and let $H^{2 k}\left(\Gamma_{c}, \alpha\right)$ be the set of all maps

$$
f: \Gamma_{c} \longrightarrow S^{k}\left(\mathfrak{g}_{\xi}^{*}\right) \text {. }
$$

The sum

$$
H\left(\Gamma_{c}, \alpha\right)=\bigoplus H^{2 k}\left(\Gamma_{c}, \alpha\right)
$$

is a graded ring under point-wise multiplication and we will define a morphism of graded rings

$$
\mathcal{K}_{c}: H(\Gamma, \alpha) \longrightarrow H\left(\Gamma_{c}, \alpha\right)
$$

as follows: For $e \in \Gamma_{c}$ let $p$ and $q$ be the vertices of $e$. The projection $\mathfrak{g}^{*} \longrightarrow \mathfrak{g}_{e}^{*}$ maps $\mathfrak{g}_{\xi}^{*}$ bijectively onto $\mathfrak{g}_{e}^{*}$ since $\alpha_{p, e}(\xi) \neq 0$, so one has a composite map

$$
\mathfrak{g}^{*} \longrightarrow \mathfrak{g}_{e}^{*} \leftrightarrow \mathfrak{g}_{\xi}^{*}
$$

and hence an induced morphism of graded rings:

$$
S\left(\mathfrak{g}^{*}\right) \longrightarrow S\left(\mathfrak{g}_{e}^{*}\right) \leftrightarrow S\left(\mathfrak{g}_{\xi}^{*}\right) .
$$

If $f$ is in $H(\Gamma, \alpha)$, the images of $f_{p}$ and $f_{q}$ in $S\left(\mathfrak{g}_{e}^{*}\right)$ are the same by (1.21) and hence so are their images in $S\left(\mathfrak{g}_{\xi}^{*}\right)$. We define $\mathcal{K}_{c}(f)(e)$ to be this common image and call the map $\mathcal{K}_{c}$ the Kirwan map.

Next we will define a morphism, $\gamma_{c}$, of $S\left(\mathfrak{g}_{\xi}^{*}\right)$-modules, mapping $H\left(\Gamma_{c}, \alpha\right)$ into the quotient field of $S\left(\mathfrak{g}_{\xi}^{*}\right)$. To define $\gamma_{c}$, let $e$, as above, be an element of $\Gamma_{c}$ and let $p$ and $q$ be the vertices of $e$. We will assume that $\phi(p)>c$ and $\phi(q)<c$ and, hence, that $\alpha_{q, e}(\xi)>0$. Let

$$
e_{i}^{+}, \quad i=1, \ldots, d-1
$$

be the other edges of $\Gamma$ (other than $e$ ) intersecting at $p$ and

$$
e_{i}^{-}, \quad i=1, \ldots, d-1
$$

be the other edges of $\Gamma$ intersecting at $q$. By the compatibility axiom we can assume that $\alpha_{p, e_{i}^{+}}$and $\alpha_{q, e_{i}^{-}}$have the same image in $\mathfrak{g}_{e}^{*}$ and, hence, under the identification, $\mathfrak{g}_{e}^{*} \leftrightarrow \mathfrak{g}_{\xi}^{*}$, have the same image in $\mathfrak{g}_{\xi}^{*}$. This implies that

$$
\alpha_{i, e}^{\#}=: \alpha_{p, e_{i}^{+}}-m_{i, e}^{+} \alpha_{p, e}=\alpha_{q, e_{i}^{-}}-m_{i, e}^{-} \alpha_{q, e}
$$

where

$$
m_{i, e}^{+}=\frac{\alpha_{p, e_{i}^{+}}(\xi)}{\alpha_{p, e}(\xi)} \quad \text { and } \quad m_{i, e}^{-}=\frac{\alpha_{q, e_{i}^{-}}(\xi)}{\alpha_{q, e}(\xi)}
$$


Let

$$
m_{e}=\alpha_{q, e}(\xi)=-\alpha_{p, e}(\xi)
$$

and note that, since $\phi(p)>\phi(q), m_{e}=\left|m_{e}\right|>0$.

We now define, for $f \in H^{2 k}\left(\Gamma_{c}, \alpha\right)$,

$$
\gamma_{c} f=\sum_{e \in \Gamma_{c}} \frac{1}{m_{e}} \frac{f(e)}{\prod_{i} \alpha_{i, e}^{\#}}
$$

and we define $\mathfrak{p}_{c}$ to be the composition

$$
\mathfrak{p}_{c}=\gamma_{c} \mathcal{K}_{c}
$$

TheOREM 2.3. $\mathfrak{p}_{c}$ is a map of $H^{2 k}(\Gamma, \alpha)$ into $S^{k-d+1}\left(\mathfrak{g}_{\xi}^{*}\right)$.

We will prove this by obtaining an explicit "residue formula" for $\mathfrak{p}_{c}(f), f \in$ $H^{2 k}(\Gamma, \alpha)$. This residue formula can be viewed as a kind of combinatorial version of the Jeffrey-Kirwan theorem described in $\S 1.3$. It is also closely related to the localization theorem proved by Jaap Kalkman and the first author in [GK], and the residue results which we will describe in the next section are mostly taken from [GK].

2.6. Residues. Let $\alpha_{1}, \ldots \alpha_{d}$ be elements of $\mathfrak{g}^{*}$ and $\xi$ be an element of $\mathfrak{g}$ with the property that $\alpha_{i}(\xi) \neq 0$ for all $i$. Given $f \in S^{k}\left(\mathfrak{g}^{*}\right)$ we define an element

$$
\operatorname{Res}_{\xi} \frac{f}{\prod \alpha_{i}}
$$

of $S^{k-d+1}\left(\mathfrak{g}_{\xi}^{*}\right)$ as follows: Choose a basis $x, y_{1}, . ., y_{n-1}$ of $\mathfrak{g}^{*}$ such that $y_{1}, . ., y_{n-1}$ is a basis of $\mathfrak{g}_{\xi}^{*}$ and $x(\xi)=1$. Let

$$
\alpha_{i}=m_{i} x-\sum_{j=1}^{n-1} a_{i j} y_{j}
$$

and let

$$
f(x)=\sum_{r=0}^{k} x^{r} f_{r}(y)
$$

and

$$
\beta_{i}=m_{i}^{-1} \sum a_{i j} y_{j}
$$

then

$$
\frac{f}{\alpha_{1} \ldots \alpha_{d}}=\left(\prod m_{i}\right)^{-1} x^{-d} \sum x^{r} f_{r}\left(1-\frac{\beta_{1}}{x}\right)^{-1} \cdots\left(1-\frac{\beta_{d}}{x}\right)^{-1} .
$$

Now replace $\left(1-\frac{\beta_{i}}{x}\right)^{-1}$ by the power series

$$
\sum_{k=0}^{\infty} x^{-k} \beta_{i}^{k}
$$


and define (2.19) to be the coefficient of $x^{-1}$ in the product on the right. It is easy to see that this definition doesn't depend on the choice of $x, y_{1}, \ldots, y_{n}$. If the $\alpha_{i}$ 's are pairwise linearly independent (i.e., if, for $i \neq j, \alpha_{i}$ and $\alpha_{j}$ are not multiples of each other) there is a relatively simple formula for (2.19).

Lemma 1. Let $A$ be a graded commutative algebra over $\mathbb{C}$ and let $f=f(x)$ be a polynomial in $x$ with coefficients in $A$. Then for indeterminants $z_{1}, \ldots, z_{d}$

$$
\operatorname{Res}_{x} \frac{f(x)}{\left(x-z_{1}\right) \cdots\left(x-z_{d}\right)}=\sum_{i=1}^{d} \frac{f\left(z_{i}\right)}{\prod_{j \neq i}\left(z_{i}-z_{j}\right)} .
$$

Proof. The conclusion follows immediately from the decomposition in simple fractions

$$
\frac{f(x)}{\left(x-z_{1}\right) \cdots\left(x-z_{d}\right)}=F(x)+\sum_{i=1}^{d} \frac{f\left(z_{i}\right)}{\prod_{j \neq i}\left(z_{i}-z_{j}\right)} \frac{1}{x-z_{i}}
$$

where $F(x)$ is a polynomial term in $x$.

Let

$$
h=\frac{f}{\prod_{j=1}^{d}\left(x-z_{j}\right)} \quad \text { and } \quad h_{j}=\frac{f\left(z_{j}\right)}{\prod_{r \neq j}\left(z_{j}-z_{r}\right)}, \forall j .
$$

LEMMA 2. $h \in A[x]$ if and only if $\operatorname{Res}_{x}\left(x^{k} h\right)=0, \forall k \geq 0$.

Proof. From (2.22) we get that

$$
\operatorname{Res}_{x}\left(x^{k} h\right)=\sum_{j=1}^{d}\left(z_{j}\right)^{k} h_{j}
$$

Then the fact that $\operatorname{Res}_{x}\left(x^{k} h\right)=0, \forall k=1, \ldots, d$ can be written as

$$
\left(\begin{array}{ccccc}
z_{1}^{1} & \cdots & z_{j}^{1} & \cdots & z_{d}^{1} \\
\vdots & & \vdots & & \vdots \\
z_{1}^{k} & \cdots & z_{j}^{k} & \cdots & z_{d}^{k} \\
\vdots & & \vdots & & \vdots \\
z_{1}^{d} & \cdots & z_{j}^{d} & \cdots & z_{d}^{d}
\end{array}\right)\left(\begin{array}{c}
h_{1} \\
\vdots \\
h_{j} \\
\vdots \\
h_{d}
\end{array}\right)=0 .
$$

Since the corresponding Vandermonde determinant is non-zero we deduce that $h_{1}=$ $\cdots=h_{d}=0$, i.e. $f\left(z_{j}\right)=0, \forall j=1, \ldots, d$, from which we obtain that $h \in A[x]$; the other implication is clear.

We will now apply lemma 1 to the evaluation of (2.19). Let

$$
m_{i}=\alpha_{i}(\xi)
$$


and for $i \neq j$ let

$$
\alpha_{j, i}^{\#}=\alpha_{j}-m_{j, i} \alpha_{i}
$$

with

$$
m_{j, i}=\frac{\alpha_{j}(\xi)}{m_{i}}
$$

Note that $\alpha_{j, i}^{\#} \in \mathfrak{g}_{\xi}^{*}$ since $\alpha_{j, i}^{\#}(\xi)=\alpha_{j}(\xi)-\alpha_{j}(\xi)=0$. Let

$$
\mathfrak{g}_{i}^{*}=\mathfrak{g}^{*} /\left\{c \alpha_{i} ; c \in \mathbb{R}\right\}
$$

The projection map: $\mathfrak{g}^{*} \longrightarrow \mathfrak{g}_{i}^{*}$ is bijective on $\mathfrak{g}_{\xi}^{*}$, so one gets a composite map

$$
\mathfrak{g}^{*} \longrightarrow \mathfrak{g}_{i}^{*} \stackrel{\simeq}{\longrightarrow} \mathfrak{g}_{\xi}^{*}
$$

as in $\S 2.5$ and hence a ring morphism

$$
\mathcal{K}_{i}: S\left(\mathfrak{g}^{*}\right) \longrightarrow S\left(\mathfrak{g}_{\xi}^{*}\right)
$$

Theorem 2.4. For $f \in S^{k}\left(\mathfrak{g}^{*}\right)$

$$
\operatorname{Res}_{\xi} \frac{f}{\alpha_{1} \ldots \alpha_{d}}=\sum_{i} \frac{1}{m_{i}} \frac{\mathcal{K}_{i} f}{\prod_{j \neq i} \alpha_{j, i}^{\#}} .
$$

Proof. With the notations (2.20)-(2.21)

$$
\frac{f}{\prod \alpha_{i}}=\left(\prod m_{k}\right)^{-1} \frac{f(x, y)}{\prod\left(x-\beta_{k}(y)\right)} .
$$

Thus by Lemma 1

$$
\begin{aligned}
\operatorname{Res}_{\xi} \frac{f}{\prod \alpha_{i}} & =\left(\prod m_{k}\right)^{-1} \sum_{i} \frac{f\left(\beta_{i}, y\right)}{\prod_{k \neq i}\left(\beta_{i}-\beta_{k}\right)} \\
& =\sum_{i} \frac{1}{m_{i}} \frac{f\left(\beta_{i}, y\right)}{\prod_{k \neq i} m_{k}\left(\beta_{i}-\beta_{k}\right)} .
\end{aligned}
$$

But $m_{k}\left(\beta_{i}-\beta_{k}\right)=\alpha_{k, i}^{\#}$ and the map

$$
\mathcal{K}_{i}: S\left(\mathfrak{g}^{*}\right) \longrightarrow S\left(\mathfrak{g}_{\xi}^{*}\right)
$$

maps $x$ to $\beta_{i}$ and $y_{k}$ to itself, so $\mathcal{K}_{i} f(x, y)=f\left(\beta_{i}, y\right)$. Thus the sum on the right is identical with the right hand side of $(2.22)$. 
2.7. The Jeffrey-Kirwan theorem. We will prove Theorem 2.3 by deducing it from the following result:

Theorem 2.5. For $f \in H^{2 k}(\Gamma, \alpha)$

$$
\mathfrak{p}_{c}(f)=\sum_{\phi(p)<c} \operatorname{Res}_{\xi} \frac{f_{p}}{\prod \alpha_{p, e}} .
$$

In particular, $\mathfrak{p}_{c}(f)$ is in $S^{k-d+1}\left(\mathfrak{g}_{\xi}^{*}\right)$.

Proof. Choose $c=c_{0}>c_{1}>c_{2}>\ldots>c_{N}$ in $\mathbb{R}-\phi(V)$ so that $\phi(V) \subset\left(c_{N}, \infty\right)$ and $\forall r \geq 0$ there is exactly one vertex $p_{r}$ with $\phi\left(p_{r}\right) \in\left(c_{r+1}, c_{r}\right)$. Inspection of $(2.18)$ shows that

$$
\mathfrak{p}_{c_{r}}(f)-\mathfrak{p}_{c_{r+1}}(f)=\sum \frac{1}{m_{i}} \frac{\mathcal{K}_{i} f}{\prod_{j \neq i} \alpha_{j, i}^{\#}},
$$

where $e_{i}, i=1, \ldots, d$, are the edges of $\Gamma$ containing $p_{r}$ and $\alpha_{i}=\alpha_{p_{r}, e_{i}}$. On the other hand, by Theorem 2.4, the right hand side of (2.25) is just

$$
\operatorname{Res}_{\xi} \frac{f\left(p_{r}\right)}{\prod \alpha_{p_{r}, e}} .
$$

The conclusion follows since $\mathfrak{p}_{c_{N}}(f)=0$.

Corollary 2.1. If $\pi_{*}$ is the map given by (2.9) then, for $f \in H^{2 k}(\Gamma, \alpha)$,

$$
\operatorname{Res}_{\xi}\left(\pi_{*} f\right)=0 \text {. }
$$

We conclude this section by observing that combining corollary 2.1 and lemma 2 we obtain a new proof of theorem 2.2 for graphs that satisfy the no-cycle condition:

Let $f \in H^{2 k}(\Gamma, \alpha)$. Then, as in (2.10),

$$
\pi_{*} f=\frac{g}{\prod_{j=1}^{N} \alpha_{j}},
$$

where $g \in S^{k-d+N}\left(\mathfrak{g}^{*}\right)$ and $\alpha_{1}, \cdots, \alpha_{N}$ are pair-wise linearly independent.

Let $\xi$ generate an orientation of $\Gamma$ with no cycles and choose $\theta \in \mathfrak{g}^{*}$ such that $\theta(\xi)=1$ and $\theta$ is not equal to any of $\alpha_{1}, \cdots, \alpha_{N}$. Then $\theta^{r} f \in H^{2(k+r)}(\Gamma, \alpha)$ and

$$
\pi_{*}\left(\theta^{r} f\right)=\frac{\theta^{r} g}{\prod_{i=1}^{N} \alpha_{i}} .
$$

But (2.26) implies that $\operatorname{Res}_{\xi}\left(\pi_{*}\left(\theta^{r} f\right)\right)=0 \forall r \geq 0$ and it follows now from lemma 2 that $\pi_{*} f \in S^{k-d}\left(\mathfrak{g}^{*}\right)$.

2.8. The Betti numbers of the pair $(\Gamma, \alpha)$. For $\xi \in \mathcal{P}$ and $p \in V_{\Gamma}$, let $\sigma_{p}=\sigma_{p}(\xi)$ be the number of edges $e$, with one vertex $p$, for which $\alpha_{p, e}(\xi)<0$. Let $\beta_{k}$ be the number of vertices $p$ with $\sigma_{p}=k$. Since $\sigma_{p}$ depends on $\xi$, it is surprising to find that these "Betti numbers" don't. 
TheOREM 2.6. The $\beta_{k}$ 's don't depend on $\xi ;$ i.e. they are combinatorial invariants of $(\Gamma, \alpha)$.

Proof. Let $\mathcal{P}_{i}, i=1, \ldots, N$, be the connected components of $\mathcal{P}$ and consider an $(n-1)$-dimensional wall separating two adjacent $\mathcal{P}_{i}$ 's. This wall is defined by an equation of the form

$$
\alpha_{p, e}(\xi)=0
$$

for some $(p, e) \in I_{\Gamma}$. Let $q$ be the other vertex of $e$ and lets compute the changes in $\sigma_{p}$ and $\sigma_{q}$ as $\xi$ passes through this wall: Let $e_{i}, i=1, \ldots, d$ be the edges meeting at $p$ and $e_{i}^{\prime}, i=1, \ldots, d$ be the edges meeting at $q$ ( with $e_{d}=e_{d}^{\prime}=e$ ). By (1.18) we can order the $e_{i}$ 's so that, for $i \leq d-1$,

$$
\alpha_{p, e_{i}}=\alpha_{q, e_{i}^{\prime}}+c_{i} \alpha_{p, e} .
$$

From (1.17) follows that for every $i=1, \ldots, d-1$,

$$
\operatorname{dim}\left(\operatorname{ker} \alpha_{p, e} \cap \operatorname{ker} \alpha_{p, e_{i}}\right)=n-2 .
$$

Therefore there exists $\xi_{0}$ such that $\alpha_{p, e}\left(\xi_{0}\right)=0$ but $\alpha_{p, e_{i}}\left(\xi_{0}\right)=\alpha_{q, e_{i}^{\prime}}\left(\xi_{0}\right) \neq 0$, for all $i=1, \ldots, d-1$.

Then there exists a neighborhood $U$ of $\xi_{0}$ in $\mathfrak{g}$ such that for all $i=1, \ldots, d-1$ and $\xi \in U, \alpha_{p, e_{i}}(\xi)$ and $\alpha_{q, e_{i}^{\prime}}(\xi)$ have the same sign and this common sign doesn't depend on $\xi \in U$. Such a neighborhood will intersect both regions created by the wall (2.27). Now suppose that $\xi \in U$ and that $r$ of the numbers $\alpha_{p, e_{i}}(\xi), i=1, \ldots, d-1$, are negative. Since $\alpha_{p, e}(\xi)=-\alpha_{q, e}(\xi)$, it follows that for $\alpha_{p, e}(\xi)$ positive

$$
\sigma_{p}=r \quad \text { and } \quad \sigma_{q}=r+1
$$

and for $\alpha_{p, e}(\xi)$ negative

$$
\sigma_{p}=r+1 \quad \text { and } \quad \sigma_{q}=r
$$

In either case, as $\xi$ passes through the wall (2.27), the Betti numbers don't change.

(For this simple and beautiful proof of the well-definedness of the Betti numbers we are indebted to Ethan Bolker.)

2.9. Betti numbers and cohomology. Simple examples show that the formula (1.35) is not true for an arbitrary graph-axial function pair $(\Gamma, \alpha)$. However, we will prove that if $(\Gamma, \alpha)$ has the no-cycle property for some $\xi \in \mathcal{P}$ then the equality (1.35) can be replaced by the inequality

$$
\operatorname{dim} H^{2 k}(\Gamma, \alpha) \leq \sum \beta_{r} \operatorname{dim} S^{k-r}\left(\mathfrak{g}^{*}\right) .
$$

In addition we will show that, for $k$ large,

$$
\operatorname{dim} H^{2 k}(\Gamma, \alpha)=\sum \beta_{r} \operatorname{dim} S^{k-r}\left(\mathfrak{g}^{*}\right)+O\left(k^{n-3}\right) .
$$

(Note that since

$$
\operatorname{dim} S^{k}\left(\mathfrak{g}^{*}\right)=\left(\begin{array}{c}
k+n-1 \\
n-1
\end{array}\right)=\frac{1}{(n-1) !}\left(k^{n-1}+\left(\begin{array}{c}
n \\
2
\end{array}\right) k^{n-2}+O\left(k^{n-3}\right)\right)
$$


the first term on the right hand side is strictly greater than the error term.) In particular, if $n=2$, the formula (2.29) asserts that

$$
\operatorname{dim} H^{2 k}(\Gamma, \alpha)=\sum \beta_{r} \operatorname{dim} S^{k-r}\left(\mathfrak{g}^{*}\right)
$$

for all $k$ greater than some fixed $k_{0}$.

Proof. Let $\alpha_{i} \in \mathfrak{g}^{*}, i=1, \ldots, N$, be a pairwise linearly independent set of vectors with the property that every one of the vectors $\alpha_{p, e},(p, e) \in I_{\Gamma}$, is a multiple of a vector in this set. Let $I$ be the graded ideal in $S\left(\mathfrak{g}^{*}\right)$ generated by the monomials

$$
g_{i}=\alpha_{1} \cdots \widehat{\alpha_{i}} \cdots \alpha_{N} .
$$

LEMMA 3. The algebraic dimension of the quotient ring $S\left(\mathfrak{g}^{*}\right) / I$ is $n-2$.

Proof. This follows trivially from the fact that the algebraic variety defined by $I$ is the union of the sets $\alpha_{i}=\alpha_{j}=0, i \neq j$.

As a corollary of this lemma we get the bound

$$
\operatorname{dim} S^{k}\left(\mathfrak{g}^{*}\right) / I^{k}=O\left(k^{n-3}\right) .
$$

Now let $\phi: V_{\Gamma} \longrightarrow \mathbb{R}$ be a function which is positively oriented with respect to $\xi$ and let $H_{c}(\Gamma, \alpha)$ be the subring of $H(\Gamma, \alpha)$ consisting of all maps $f: V_{\Gamma} \longrightarrow S\left(\mathfrak{g}^{*}\right)$ with support on the set $\phi \geq c$. Let $p \in V_{\Gamma}$ with $\phi(p)=c$ and suppose that there are no points $q \in V_{\Gamma}$ with $\phi(q)$ on the interval $\left(c, c^{\prime}\right)$. Letting $\sigma_{p}=r$ we will prove the Morse inequality

$$
\operatorname{dim} H_{c}^{2 k}(\Gamma, \alpha)-\operatorname{dim} H_{c^{\prime}}^{2 k}(\Gamma, \alpha) \leq \operatorname{dim} S^{k-r}\left(\mathfrak{g}^{*}\right)
$$

and an inequality in the opposite direction:

$$
\operatorname{dim} H_{c}^{2 k}(\Gamma, \alpha)-\operatorname{dim} H_{c^{\prime}}^{2 k}(\Gamma, \alpha) \geq \operatorname{dim} I^{k-r} .
$$

To prove (2.32) let $e_{i}, i=1, \ldots, d$, be the edges of $\Gamma$ containing $p$ and let $\alpha_{i}=\alpha_{p, e_{i}}$. We will order the $\alpha_{i}$ 's so that $\alpha_{i}(\xi)<0$ for $1 \leq i \leq r$ and $\alpha_{i}(\xi)>0$ for $r+1 \leq i \leq d$. Then if $f \in H_{c}^{2 k}(\Gamma, \alpha), f(p)$ must be a multiple of $\alpha_{1}, \ldots, \alpha_{r}$ so the image of the restriction map

$$
H_{c}^{2 k}(\Gamma, \alpha) \longrightarrow S^{k}\left(\mathfrak{g}^{*}\right), \quad f \longrightarrow f(p)
$$

is contained in $S^{k-r}\left(\mathfrak{g}^{*}\right) \alpha_{1} \cdots \alpha_{r}$. Since the kernel of this map is $H_{c^{\prime}}^{2 k}(\Gamma, \alpha)$, this proves (2.32). We will prove the inequality (2.33) by showing that if $h \in I^{k-r}$ then $h \alpha_{1} \cdots \alpha_{r}$ is in the image of (2.34). Indeed, if $h \in I^{k-r}$ then $h \alpha_{1} \cdots \alpha_{r}$ can be written as a sum

$$
\sum_{i=r+1}^{N} h_{i} \alpha_{1} \cdots \widehat{\alpha_{i}} \cdots \alpha_{N}
$$

Let $p_{j}$ be the vertex joined to $p$ by $e_{j}$ for $j=r+1, \cdots, d$, and, for fixed $j_{0} \in$ $\{r+1, \ldots, d\}$, define $f: V_{\Gamma} \longrightarrow S^{k}\left(\mathfrak{g}^{*}\right)$ to be the map which takes the value $h \alpha_{1} \cdots \alpha_{r}$ 
at $p$, the value $h_{j_{0}} \alpha_{1} \cdots \widehat{\alpha_{j_{0}}} \cdots \alpha_{N}$ at $p_{j_{0}}$ and zero elsewhere. It is easily checked that $f \in H_{c}^{2 k}(\Gamma, \alpha)$ and $f(p)=h \alpha_{1} \cdots \alpha_{r}$. This proves (2.33).

Next let $c$ and $c^{\prime}$ be any pair of real numbers with $c<c^{\prime}$. From (2.32)-(2.33) one gets, by a simple induction, the Morse inequalities

$$
\operatorname{dim} H_{c}^{2 k}(\Gamma, \alpha)-\operatorname{dim} H_{c^{\prime}}^{2 k}(\Gamma, \alpha) \leq \sum \beta_{r}\left(c, c^{\prime}\right) \operatorname{dim} S^{k-r}\left(\mathfrak{g}^{*}\right)
$$

and

$$
\operatorname{dim} H_{c}^{2 k}(\Gamma, \alpha)-\operatorname{dim} H_{c^{\prime}}^{2 k}(\Gamma, \alpha) \geq \sum \beta_{r}\left(c, c^{\prime}\right) \operatorname{dim} I^{k-r},
$$

where $\beta_{r}\left(c, c^{\prime}\right)$ is the number of points $p \in V_{\Gamma}$ with $\sigma_{p}=r$ and $c \leq \phi(p)<c^{\prime}$. In particular, for $c^{\prime}>>0$ and $c<<0$, one gets from these estimates and from (2.31) the inequalities (2.28) and (2.29).

2.10. The role of the zeroth Betti number. An example of a graph-axial function pair that fails to satisfy (1.35) is the $d=n=2$ example described at the end of $\S 2.1$. The graph in this example is a connected graph; so its topological zeroth Betti number is 1 . However, its graph theoretical zeroth Betti number, $\beta_{0}$, is $N$. A simple computation shows that, for this example, the identity (1.35) holds for all $k>0$. But for $k=0$ the left hand side of (1.35) is 1 (since the graph is connected) whereas the right hand side, $\beta_{0}$, is $N$. From this example one can generate examples of graph-axial function pairs, $(\Gamma, \alpha)$, for which the estimate (2.29) is "best possible" by taking Cartesian products of this graph with graphs which do satisfy (1.35).

However, by making some additional assumptions on the pair $(\Gamma, \alpha)$ one can considerably improve (2.29). The assumptions we will make are of two kinds:

1. To avoid the problem posed by the example we have just described, we will assume that the zeroth Betti numbers of certain connected subgraphs of $\Gamma$ are equal to 1 .

2. For every $p \in V_{\Gamma}$ we will make certain "general position" hypothesis about the vectors $\alpha_{p, e},(p, e) \in I_{\Gamma}$. To formulate these hypothesis we introduce the following refinement of the notion of "pairwise linearly independent":

Definition. A collection of vectors $\alpha_{i} \in \mathfrak{g}^{*}, i=1, \ldots, N$, is l-independent if, for every sequence $1 \leq i_{1}<i_{2}<\ldots<i_{l} \leq N$, the vectors $\alpha_{i_{1}}, \ldots, \alpha_{i_{l}}$ are linearly independent.

Now let $(\Gamma, \alpha)$ be a graph-axial function pair which satisfies the no-cycle condition for some $\xi \in \mathcal{P}$. The main result of this section is the following sharpening of (2.29):

\section{THEOREM 2.7. Suppose the following hypotheses hold:}

1. For every subspace $\mathfrak{h}$ of $\mathfrak{g}$ of codimension strictly less than l, the zeroth Betti number of the connected components of $\Gamma_{\mathfrak{h}}$ are equal to 1 .

2. For every vertex $p$ of $\Gamma$, the vectors $\alpha_{p, e},(p, e) \in I_{\Gamma}$, are l-independent.

Then:

$$
\operatorname{dim} H^{2 k}(\Gamma, \alpha)=\sum \beta_{r} \operatorname{dim} S^{k-r}\left(\mathfrak{g}^{*}\right)+O\left(k^{n-1-l}\right) .
$$


REMARK. For $l=2$ the above conditions are always satisfied; (2.29) is the particular case of $(2.35)$ corresponding to $l=2$.

For $l=n$ this theorem says that the left hand side of (2.35) is equal to the first term on the right for $k$ greater than some fixed $k_{0}$. This result can be slightly improved.

THEOREM 2.8. If the hypotheses of theorem 2.7 hold for $l=n$ then

$$
\operatorname{dim} H^{2 k}(\Gamma, \alpha)=\sum \beta_{r} \operatorname{dim} S^{k-r}\left(\mathfrak{g}^{*}\right)
$$

for $k>d-n$.

We will prove these two results by refining the Morse inequalities (2.33). For this we will need the following generalization of Lemma 3 of $\S 2.9$ :

LEMMA 4. Let $\gamma_{1}, \ldots, \gamma_{N}$ be a collection of vectors in $\mathfrak{g}^{*}$ which are l-independent and let $I_{l}$ be the ideal in $S\left(\mathfrak{g}^{*}\right)$ generated by the monomials

$$
\frac{\gamma_{1} \cdots \gamma_{N}}{\gamma_{i_{1}} \cdots \gamma_{i_{l-1}}}, \quad 1 \leq i_{1}<\cdots i_{l-1} \leq N
$$

Then the algebraic dimension of $S\left(\mathfrak{g}^{*}\right) / I_{l}$ is $n-l$. Moreover, if $N \geq n$ and $n=l$ then $S^{m}\left(\mathfrak{g}^{*}\right)=I_{l}^{m}$ for $m>N-n$.

(For the proof of this lemma see the appendix at the end of this section.)

Proof of theorem 2.7. Let $\phi: V_{\Gamma} \longrightarrow \mathbb{R}$ be a strictly monotone function which is positively oriented with respect to $\xi$. Let $p$ be an arbitrary vertex of $\Gamma$, let $c=\phi(p)$ and assume that there are no points $q \in V_{\Gamma}$ with $c<\phi(q)<c^{\prime}$. Let $e_{1}, \ldots, e_{d}$ be the edges of $\Gamma$ containing $p$ and let $\alpha_{i}=\alpha_{p, e_{i}}$. We can order these vectors so that $\alpha_{i}(\xi)<0$ for $1 \leq i \leq r$ and $\alpha_{i}(\xi)>0$ for $r+1 \leq i \leq d$, where $r=\sigma_{p}$. Let $N=d-r$.

Suppose $l \leq N$. We will prove that

$$
\operatorname{dim} H_{c}^{2 k}(\Gamma, \alpha)-\operatorname{dim} H_{c^{\prime}}^{2 k}(\Gamma, \alpha) \geq \operatorname{dim} I_{l}^{k-r},
$$

where $I_{l}$ is the ideal of $S\left(\mathfrak{g}^{*}\right)$ constructed as in lemma 4 using the $N=d-r$ vectors $\alpha_{r+1}, \ldots, \alpha_{d}$ (which are $l$-independent, by hypothesis 2$)$, i.e. $I_{l}$ is the ideal in $S\left(\mathfrak{g}^{*}\right)$ generated by the monomials

$$
\frac{\alpha_{r+1} \cdots \alpha_{d}}{\alpha_{i_{1}} \cdots \alpha_{i_{l-1}}}, \quad r+1 \leq i_{1}<\ldots<i_{l-1} \leq N
$$

To show (2.38), consider (as in $\S 2.9$ ) the restriction map

$$
H_{c}^{2 k}(\Gamma, \alpha) \longrightarrow S^{k-r}\left(\mathfrak{g}^{*}\right) \alpha_{1} \cdots \alpha_{r}
$$

given by $f \longrightarrow f(p)$. The kernel of this map is $H_{c^{\prime}}^{2 k}(\Gamma, \alpha)$; so, to prove (2.38) it suffices to show that the image of this map contains $I_{l}^{k-r} \alpha_{1} \cdots \alpha_{r}$. Consider the set of vectors $\alpha_{i_{1}}, \ldots, \alpha_{i_{l-1}}, r+1 \leq i_{1}<\ldots<i_{l-1} \leq d$. These vectors are linearly independent; so 
their annihilator, $\mathfrak{h}$, is of codimension $l-1$ in $\mathfrak{g}$. Let $\Gamma_{1}$ be the connected component of $\Gamma_{\mathfrak{h}}$ containing $p$. Since the numbers $\alpha_{i_{1}}(\xi), \ldots, \alpha_{i_{l-1}}(\xi)$ are greater than zero, $p$ is a local minimum point for the restriction of $\phi$ to $\Gamma_{\mathfrak{h}}$; so, by hypothesis $1, p$ is also a global minimum. Therefore the vertices of $\Gamma_{1}$ are contained in the set $\phi \geq c$, and, hence the Thom class, $\tau_{1}$, of $\Gamma_{1}$, is supported in this set, i.e. is an element of $H_{c}(\Gamma, \alpha)$. However, at $p, \tau_{1}$ is equal to

$$
\left(\frac{\alpha_{r+1} \cdots \alpha_{d}}{\alpha_{i_{1}} \cdots \alpha_{i_{l-1}}}\right) \alpha_{1} \cdots \alpha_{r} .
$$

This argument shows that, for all generators, $f$, of $I_{l}, f \alpha_{1} \cdots \alpha_{r}$ is in the image of the restriction map (2.39). Hence the image of this restriction map contains $I_{l}^{k-r} \alpha_{1} \cdots \alpha_{r}$, which proves $(2.38)$.

Now suppose that $l>N$. In this case we can simply take $\mathfrak{h}$ to be the annihilator of $\alpha_{r+1}, \ldots, \alpha_{d}$, and, by the same argument as above, conclude that the image of the restriction map is equal to $S^{k-r}\left(\mathfrak{g}^{*}\right) \alpha_{1} \cdots \alpha_{r}$. Hence for $l>N$,

$$
\operatorname{dim} H_{c}^{2 k}(\Gamma, \alpha)-\operatorname{dim} H_{c^{\prime}}^{2 k}(\Gamma, \alpha)=\operatorname{dim} S^{k-r}\left(\mathfrak{g}^{*}\right) .
$$

The proof of the estimate (2.35) via (2.38), (2.40) and lemma 4 is the same as the proof of the estimate (2.29) via (2.33) and lemma 3 . We will omit details.

Proof of theorem 2.8. Let $l=n$. If $N<n$ the equality (2.40) holds for all $k$ (as we have just seen). If $N \geq n$ then, by lemma 4 and by (2.38), the equality (2.40) holds if $k-r>N-n$, i.e. if $k>d-n$. Thus for $l=n,(2.35)$ can be sharpened to (2.36).

Appendix: The proof of Lemma 4. We will prove by induction that the algebraic variety defined by $I_{l}$ is the union of the sets

$$
\gamma_{i_{1}}=\cdots=\gamma_{i_{l}}=0, \quad 1 \leq i_{1}<\cdots<i_{l} \leq N .
$$

Let $x$ be a point on this variety. Since $I_{l-1} \subset I_{l}$, the variety defined by $I_{l-1}$ contains the variety defined by $I_{l}$; so it follows by induction that there exists a sequence $1 \leq i_{1}<\cdots<i_{l-1} \leq N$ with $\gamma_{i_{1}}=\cdots=\gamma_{i_{l-1}}=0$ at $x$. However, since $I_{l}$ contains the quotient of $\gamma_{1} \cdots \gamma_{N}$ by $\gamma_{i_{1}} \cdots \gamma_{i_{l-1}}$, there exists some $j \neq i_{1}, \ldots, i_{l-1}$ such that $\gamma_{j}=0$ at $x$. This proves the first assertion of lemma 4 . Now let $l=n$. We will prove that

$$
S^{m}\left(\mathfrak{g}^{*}\right)=I_{n}^{m}
$$

for $m>N-n$ by a double induction on $n$ and $N$.

The equality above is true if $N=n$ or if $n=1$, as can be easily checked. Consider now a pair $(N, n)$ with $N>n$. We now assume that the assertion is true for $(N-1, n-1)$ and for $(N-1, n)$.

Let $\mathfrak{h}$ be the annihilator of $\gamma_{N}$ in $\mathfrak{g}$. The restriction map

$$
S\left(\mathfrak{g}^{*}\right) \longrightarrow S\left(\mathfrak{h}^{*}\right)
$$

maps $\gamma_{1}, \ldots, \gamma_{N-1}$ onto vectors $\beta_{1}, \ldots, \beta_{N-1}$, which are $(n-1)$-independent in $\mathfrak{h}^{*}$; so, by induction, every element of $S^{m}\left(\mathfrak{h}^{*}\right), m>N-n$, is in the ideal generated by the 
monomials

$$
\frac{\beta_{1} \cdots \beta_{N-1}}{\beta_{i_{1}} \cdots \beta_{i_{n-2}}}, \quad 1 \leq i_{1}<\ldots<i_{n-2} \leq N-1
$$

Since the kernel of the map $S\left(\mathfrak{g}^{*}\right) \longrightarrow S\left(\mathfrak{h}^{*}\right)$ is the ideal generated by $\gamma_{N}$, it follows that for $m>N-n$, every element of $S^{m}\left(\mathfrak{g}^{*}\right)$ can be written as a linear combination of

$$
\frac{\gamma_{1} \cdots \gamma_{N-1} \gamma_{N}}{\gamma_{i_{1}} \cdots \gamma_{i_{n-2}} \gamma_{N}}, \quad 1 \leq i_{1}<\ldots<i_{n-2} \leq N-1
$$

with polynomial coefficients, plus a term of the form $f \gamma_{N}, f \in S^{m-1}\left(\mathfrak{g}^{*}\right)$. By induction the theorem is true in dimension $n$ for the vectors $\gamma_{1}, \ldots, \gamma_{N-1}$. Then $f$ is in the ideal generated by the monomials

$$
\frac{\gamma_{1} \cdots \gamma_{N-1}}{\gamma_{i_{1}} \cdots \gamma_{i_{n-1}}}, \quad 1 \leq i_{1}<\ldots<i_{n-1} \leq N-1
$$

and hence $f \gamma_{N}$ is in the ideal generated by

$$
\frac{\gamma_{1} \cdots \gamma_{N}}{\gamma_{i_{1}} \cdots \gamma_{i_{n-1}}}, \quad 1 \leq i_{1}<\ldots<i_{n-1} \leq N-1
$$

\section{REFERENCES}

[A] M. F. Atryah, Convexity and commuting Hamiltonians, Bull. London Math. Soc., 14 (1982), pp. 1-15.

[AB1] M. F. ATIYAH AND R. BotT, The moment map and equivariant cohomology, Topology, 23 (1984), pp. 1-28.

[AB2] M. F. ATIYAH AND R. BotT, A Lefschetz fixed point formula for elliptic complexes, I, Ann. of Math., 86 (1967), pp. 374-407.

[BV] N. Berline And M. Vergne, Classes caractéristiques équivariantes, C.R. Acad. Sci., Paris, 295 (1982), pp. 539-541.

[Br] M. BRION, Equivariant cohomology and equivariant intersection theory, in Representation Theory and Algebraic Geometry, Kluwer Acad. Publ., Dordrecht, 1998, pp. 1-37.

[CS] F. R. K. ChUNG AND S. STERnberg, Laplacian and Vibrational Spectra for Homogeneous Graphs, J. of Graph Theory, 16 (1992), pp. 605-627.

[GK] V. GUILlEmin AND J. KALKMAN, The Jeffrey-Kirwan localization theorem and residue operations in equivariant cohomology, Journal reine angew. Math., 470 (1996), pp. 123-140.

[GKM] M. Goresky, R. Kottwitz, and R. MacPherson, Equivariant cohomology, Koszul duality and the localization theorem, Invent. Math., 131 (1998), pp. 25-83.

[GS] V. Guillemin And S. Sternberg, Convexity properties of the moment mapping, Invent. Math., 67 (1982), pp. 491-513.

[Gu] V. GUILLEMIN, Deformations of a Hamiltonian action of a compact Lie group, in Integrable systems (Luminy 1991), Progress in Math., 115, Birkhäuser, Boston, 1993, pp. 227-233.

[JK] L. JEFFREY AND F. KIRWAN, Localization for non-abelian group actions, Topology, 34 (1995), pp. 291-327.

[Kl] A. A. KLYACHKO, Equivariant bundles on toric varieties, Math USSR Izvestiya, 35:2 (1990), pp. 337-375. 